\title{
Merchant Storage Investment in a Restructured Electricity Industry
}

\author{
Afzal S. Siddiqui, ${ }^{\mathrm{a}}$ Ramteen Sioshansi, ${ }^{\mathrm{b}}$ and Antonio J. Conejo ${ }^{\mathrm{c}}$
}

\begin{abstract}
Restructuring and liberalisation of the electricity industry creates opportunities for investment in energy storage, which could be undertaken by a profit-maximising merchant storage operator. Because such a firm is concerned solely with maximising its own profit, the resulting storage-investment decision may be socially suboptimal (or detrimental). This paper develops a bi-level model of an imperfectly competitive electricity market. The modelling framework assumes electricity-generation and storage-operations decisions at the lower level and storage investment at the upper level. Our analytical results demonstrate that a relatively high (low) amount of market power in the generation sector leads to low (high) storage-capacity investment by the profit-maximising storage operator relative to a welfare maximiser. This can result in net social welfare losses with a profit-maximising storage operator compared to a no-storage case. Moreover, there are guaranteed to be net social welfare losses with a profit-maximising storage operator if the generation sector is sufficiently competitive. Using a charge on generation ramping between off- and on-peak periods, we induce the profit-maximising storage operator to invest in the same level of storage capacity as the welfare-maximising firm. Such a ramping charge can increase social welfare above the levels that are attained with a welfare-maximising storage operator.
\end{abstract}

Keywords: Energy storage, Bi-level modelling, Market power

https://doi.org/10.5547/01956574.40.4.asid

\section{INTRODUCTION}

Recent years have seen a renaissance in the development of energy storage. Sioshansi et al. (2012) note that this interest in storage is prompted by a number of recent electricity-industry developments. One is that storage was viewed almost exclusively as an alternative to high-cost peaking generation in the 1970s, when much of the pumped hydroelectric capacity that is installed today was first built (cf. the work of EPRI (1976) as one example showing this). More recent analyses of energy storage, with the work of EPRI-DOE (2003) being a seminal example, recognise that storage can provide many services beyond avoiding the cost of installing and operating peaking generation. A second major development is the advent of restructured electricity markets, which provide trans-

a Department of Statistical Science, University College London; Department of Computer and Systems Sciences, Stockholm University; and Department of Decision Sciences, HEC Montréal.

b Corresponding author. Department of Integrated Systems Engineering, The Ohio State University, 240 Baker Systems Engineering Building, 1971 Neil Avenue, Columbus, Ohio 43210, United States of America. E-mail: sioshansi.1@osu. edu.

c Department of Integrated Systems Engineering and Department of Electrical and Computer Engineering, The Ohio State University. 
parent price signals for many of the services that energy storage can provide. Finally, Denholm et al. (2010) note that energy storage is expected to have a growing role in electric power systems as the penetration of variable renewable energy grows. As another example of this, von Hirschhausen (2014) surveys the potential role of energy storage in achieving the German energy-related policy goals underlying its so-called 'energiewende.'

Although energy storage has the potential to deliver transformative benefits in the production and consumption of electricity, it is by no means a panacea. One important issue around the impacts of energy storage involves its interactions within an imperfectly competitive market environment. This is because charging and discharging storage, and the resulting purchases or sales of energy, can affect market prices and the welfare of energy producers (i.e., electricity generators) and consumers. Sioshansi et al. (2009) demonstrate the potential for energy storage to mitigate the impacts on wholesale electricity prices of limited natural gas supplies after Hurricanes Katrina and Rita in 2005. They show that merchant-operated energy storage could have delivered net social welfare increases, assuming that the generation sector is perfectly competitive. Sioshansi (2010) employs a stylised partial-equilibrium model, in which electricity generation is perfectly competitive but wholesale prices respond to storage use, to analyse the welfare impacts of storage use. He shows that a merchant storage operator tends to under-use storage compared to welfare-maximising use, because it is profit-maximising for the firm to maintain a larger price difference between on- and off-peak periods (relative to welfare-maximising use). Using actual data from Germany, Schill and Kemfert (2011) compare cases in which storage may be owned by generators, who themselves may exert market power (à la a Nash-Cournot equilibrium). They find cases in which having storage in the market reduces welfare compared to a no-storage case. Sioshansi (2011) uses data from Texas to conduct a welfare analysis of energy storage in a market with high penetrations of wind energy. As in the work of Schill and Kemfert (2011), he shows that adding storage to an imperfectly competitive generation market can reduce social welfare compared to the no-storage case. Virasjoki et al. (2016) develop an equilibrium model for a Western European test network to study the impact of storage on ramping costs and grid congestion in the presence of a realistic level of renewable energy. They show that the overall welfare impact of storage is modest, but that it can, nevertheless, reduce ramping and congestion costs. This benefit of storage is limited, however, if storage-owning producers exert market power. Sioshansi (2014) uses a stylised equilibrium model to consider various ownership and market structures involving energy storage. He concludes that storage can be welfare diminishing (compared to a no-storage case) in the presence of strategic generating firms. Surprisingly, he finds that welfare losses that arise with strategic generating firms can be greater if storage operations are perfectly competitive. Shahmohammadi et al. (2018b,a) take a bi-level approach to modelling strategic interactions among storage, conventional generation, and renewable units. They model strategic offering behaviour by these units at the upper level, subject to a leastcost market-clearing model, which determines prices and dispatch, at the lower level.

An important limitation of these welfare analyses of energy storage is that they do not endogenise storage investment. In essence, these works all show that if a given amount of energy storage is in the system, there may be market structures under which social welfare is increased or decreased relative to a no-storage case. They do not, however, show whether investments in storage are individually rational in cases in which storage would be welfare-enhancing or -diminishing. Indeed, to our knowledge, there are only two works in the extant literature that endogenise storage investment within a market-equilibrium framework. However, neither of these works examines the welfare impacts of storage investment in an imperfectly competitive market. Nasrolahpour et al. (2016) develop a bi-level model with a profit-maximising storage investor at the upper level and 
market clearing at the lower level. Their model and case study account for uncertain renewable-energy availability and realistic generation constraints based on data from Alberta. They assume, however, a perfectly competitive generation sector and do not conduct a welfare analysis or a comparison of the merchant's decisions with those of a welfare-maximising storage operator. Thus, they do not model the full range of market imperfections that may yield welfare losses. Dvorkin et al. (2018) develop a tri-level model that includes a profit-maximising merchant storage investor. However, their focus is on using energy storage for alleviating transmission congestion. Thus, their modelling framework cannot unveil the types of market welfare impacts that ours does.

The aim of this work is to fill this important gap in the existing literature. We do this by extending the stylised equilibrium model that is proposed by Sioshansi (2014) to explore the welfare implications of storage with an imperfectly competitive generation sector. However, unlike the extant literature, we investigate not only market operations but also the storage-investment decision itself. Specifically, we posit that storage capacity is owned by either a profit-maximising standalone merchant investor or a welfare-maximising storage operator. In either case, the storage operator is a leader in the sense that it anticipates the response of the generation sector when making its storage-capacity investment. Moreover, the storage operator is distinct from both the market operator and the generation firms. Thus, we use a bi-level modelling framework, in which the lower level reflects market operations consisting of storage use and multiple symmetric generators across two time periods (off- and on-peak periods, respectively). At the upper level, we have a single storage investor, i.e., either the profit-maximising merchant or the welfare maximiser.

We find analytical solutions for the optimal storage capacity adopted by each type of investor and investigate the welfare effects of each. In particular, we prove that the welfare maximiser invests in more storage capacity than the profit-maximising merchant if the generation sector is relatively uncompetitive. This is because the welfare maximiser uses a large storage capacity to subvert the generators' strategy of withholding generation by moving energy to the on-peak period. Conversely, the profit-maximising merchant is content to profit from the high price differential that results from the generators' behaviour. It is, thus, reluctant to erode its profit by installing a large amount of storage capacity. In a more competitive industry, the welfare maximiser reduces its storage capacity to below that of the profit-maximising merchant as there is less welfare loss to mitigate from the exercise of market power by generators. With a relatively competitive generation sector the profit-maximising merchant maintains a relatively large storage capacity to increase its profit by trading a large volume of energy. The larger volume of energy transacted partially compensates for the lower price differential between on- and off-peak periods. If the generation sector is sufficiently competitive, then the behaviour of the profit-maximising merchant is actually welfare-diminishing vis-à-vis having no storage at all. This result runs contrary to those of Sioshansi (2014), who finds that there can be no welfare losses with a perfectly competitive generation sector if the storage capacity is fixed. Thus, our analysis shows that the welfare implications of energy storage is highly sensitive to representing the investment decision.

Next, we show that the profit-maximising merchant may be induced to invest in the same level of storage capacity as that of the welfare maximiser via a ramping charge on generation. The ramping charge essentially penalises generators and the storage operator for having a large difference in the off- and on-peak load. This ramping charge can have the effect of mitigating the incentives of storage and generation firms to maintain large price differences between the on- and off-peak periods. Finally, through numerical examples, we illustrate that storage investment by a profit-maximising merchant in the presence of a ramping charge may increase social welfare to above the level that is attained by a welfare maximiser. This is because the ramping charge offers 
another layer of 'control.' This added control can mitigate potential welfare losses from inefficient storage use and the withholding of capacity by generating firms.

Our work is limited in the potential value of energy storage that it captures, insomuch as we focus solely on using energy storage to shift generation loads from on- to off-peak periods. Energy storage is recognised today as having many more uses, as outlined by EPRI-DOE (2003). Eyer and Corey (2010) provide a comprehensive overview of potential market applications of energy storage and guidance on assessing the value of such uses. Eyer et al. (2005) provide a similar overview of 'non-market' applications of storage, including transmission- and distribution-level services. Nourai (2007); Nourai et al. (2008) discuss practical experience in using energy storage for distribution deferral and related services. Green and Vasilakos (2012); Mauritzen (2013); O'Dwyer and Flynn (2015) examine the use of energy storage to aid in integrating variable weather-dependent renewable energy resources into power systems.

Because our work neglects such uses of energy storage, it underestimates its potential value. The focus of our work is in examining the impacts of market imperfections on suboptimal or potentially welfare-diminishing storage investment and use. These types of issues are likely to be exacerbated if other potential uses of energy storage are considered. Thus, our work may be viewed as formative, insomuch as it lays out a framework for future analysis of the efficiency of market-based storage investment for these other applications.

The remainder of the paper is structured as follows. Section 2 lays out the basic assumptions underlying the market setting that we examine. Next, Section 3 derives equilibrium storage investment and operations and the resulting impacts of storage in a case with constant marginal generation costs. Section 4 then proposes a ramping charge as a means of incentivising the welfare-maximising level of storage investment by a profit-maximising merchant. Our main insights are illustrated via numerical examples, which are given in Section 5. Section 6 summarises our contributions and offers directions for future research. All proofs of the lemmata, propositions, and corollary are given in Appendix 7.1, which is posted on the Energy Journal website as supplementary material. We also present an extension of our model to a case with linear marginal generation costs in Appendix 7.2, which is posted on the Energy Journal website as supplementary material. Numerical results for this case are given in Section 5.2.

\section{MARKET MODEL}

We assume that there is a wholesale electricity market that operates across two time periods, $t=1,2$, which correspond to off- and on-peak periods, respectively. As in the work of Sioshansi (2014), we do not account for either uncertainty or transmission constraints. The market is assumed to consist of $N \geq 1$ symmetric profit-maximising firms with cost functions:

$$
c\left(g_{n, t}\right), n=1, \ldots, N,
$$

where generation output, $g_{n, t}$, is measured in MW. We assume that these cost functions are non-decreasing and convex, i.e., that $c^{\prime}\left(g_{n, t}\right) \geq 0$ and $c^{\prime \prime}\left(g_{n, t}\right) \geq 0, \forall g_{n, t} \geq 0$. We also define:

$$
g_{t}^{G}=\sum_{n=1}^{N} g_{n, t}
$$

to be total period- $t$ industry output.

Period- $t$ demand is represented by the inverse demand function:

$P_{t}(x)=A_{t}-Z_{t} x, t=1,2$, 
where $P_{t}(x)$ is given in $\$ / \mathrm{MW}$. Short-term electricity demand is normally relatively price-inelastic. We assume that the market includes a set of perfectly competitive (i.e., non-strategic) generators that offer their generation at marginal cost. This competitive fringe provides for the demand elasticity in the inverse demand functions (i.e., the demand functions can be viewed as residual demand functions that account for the competitive supply). For the demand to be both well defined and well behaved, we assume that $A_{1}, A_{2}>c^{\prime}(0)$ and $Z_{1}, Z_{2}>0$. Moreover, we assume that the period-2 demand function is always greater than the period- 1 demand function, which fits the definition of the two periods being on- and off-peak, respectively. This assumption requires that $A_{2}>A_{1}$ and $A_{2} / Z_{2}>A_{1} / Z_{1}$.

The system is assumed to have a single storage operator, which is either a profit-maximising merchant or a welfare maximiser. The storage operator must invest in storage capacity if it wishes to use storage (i.e., we assume that the system begins with no storage installed). We let $k$ represent the amount of storage capacity installed, which is measured in MW of discharging capacity. Storage investment is assumed to incur a cost of:

$$
\frac{1}{2} I k^{2}
$$

where $I>0$. The investment cost of energy storage is highly technology- and case-specific. Moreover, many technologies may face resource limits or other bounds on their capacity (e.g., reservoir size in a pumped hydroelectric storage plant or limited rare-earth metals for certain types of batteries). Our assumed quadratic investment-cost function reflects such restrictions through an increasing marginal cost. Our findings will be qualitatively similar in the presence of constant marginal cost, however.

The storage technology has a fixed roundtrip efficiency of $E \in(0,1]$. If the storage operator invests in storage it can buy $d / E$ MW during period 1 (i.e., the off-peak period), which is charged into storage. This allows the storage operator then to discharge $d$ MW during period 2 (i.e., the onpeak period), which is sold in the wholesale market. The amount of energy charged and discharged is restricted by the installed capacity, meaning that we impose the constraint $0 \leq d \leq k$. For notational convenience, we define $F=1 / E$ and note that $F \geq 1$.

Our assumption of a single storage operator allows us to model a bounding range of extreme opposite market outcomes. A single welfare-maximising storage operator represents the best possible outcome in terms of market efficiency. Conversely, a single profit-maximising storage operator may represent the worst-case market outcome, insomuch as this firm would behave as a monopolist with regards to storage investment and operation. Because an actual electricity market likely has multiple storage operators, the market outcome would likely lie between the two cases that we examine.

The market interaction is assumed to consist of two decision stages. The second stage consists of the storage operator and generating firms competing with one another by simultaneously determining charging and discharging levels and production quantities in the off- and on-peak periods. In the first stage, the storage operator determines its investment level, fully taking into account the subsequent decisions in the operating stage and the impact of its investment decision thereon. Given the sequential nature of the market interaction, we seek a subgame-perfect Nash equilibrium. Thus, we begin our analysis by first examining the Nash-Cournot equilibrium between the storage operator and generating firms in the second decision stage. Once the operating-stage equilibrium is determined, we then determine the optimal first-stage investment decision of the storage operator, while taking account of its impact on operating-stage decisions. 
Before deriving market equilibria in detail, we first show that the equilibrium production decisions of the generating firms in the two periods are symmetric (due to the assumed symmetry of the firms). To do this, we note that generating firm $n$ solves the profit-maximisation problem:

$$
\max _{g_{n, 1}, g_{n, 2}} P_{1}\left(g_{1}^{G}-F d\right) g_{n, 1}-c\left(g_{n, 1}\right)+P_{2}\left(g_{2}^{G}+d\right) g_{n, 2}-c\left(g_{n, 2}\right)
$$

S.t. $\quad g_{n, 1}, g_{n, 2} \geq 0$,

to determine its production levels. The inverse demand functions in the two periods take account of the effect of generator-production decisions, as well as charging and discharging decisions by the storage operator. The following lemma shows that the production levels of the generators in the two periods are symmetric in an equilibrium.

Lemma 1 The equilibrium production levels of the symmetric generators in the two periods are symmetric (i.e., $g_{n, 1}=g_{m, 1}$ and $g_{n, 2}=g_{m, 2}, \forall n, m=1, \ldots, N$ ).

Proof. See Appendix 7.1.

Because of this symmetry, we hereafter define the equilibrium production levels of the generating firms as:

$$
g_{n, t}=\frac{g_{t}^{G}}{N}, \forall n=1, \ldots, N, t=1,2
$$

The symmetry assumption is needed to derive closed-form analytic expressions for the generation equilibrium (cf. Section 3.1). In some cases a power system may have symmetric generation firms. Examples include Brazil, Norway, and Québec, which have electricity sectors that are dominated by hydroelectricity, and California, which has natural gas-fired generation setting the margin in most hours. Heterogeneity in generation costs would give a merit order that is increasing, which is what we investigate with the case of linear marginal costs (cf. Section 5.2 and Appendix 7.2). That being said, we do not believe that heterogeneous costs would qualitatively affect our findings in any marked way.

\section{MARKET EQUILIBRIA WITH CONSTANT MARGINAL GENERATION COSTS}

This section derives market equilibria and presents the results of our welfare analysis for the case in which the symmetric generating firms have constant marginal costs. Thus, we assume that generator costs are given by:

$$
c\left(g_{n, t}\right)=B g_{n, t}, n=1, \ldots, N,
$$

where $B>0$. Note that with constant marginal costs our previous assumption that $A_{1}, A_{2}>c^{\prime}(0)$ simplifies to $A_{2}>A_{1}>B$.

We proceed with the analysis in this section by first deriving closed-form expressions for the equilibrium production levels of the generating firms. We then determine equilibrium storage-operation and -investment decisions for the profit- and welfare-maximising storage operators, respectively. We finally compare the welfare outcomes of the two types of equilibria (i.e., with profit- and welfare-maximising storage operators). 


\subsection{Generator Equilibrium}

With constant marginal generation costs, generator $n$ 's profit-maximisation problem simplifies to:

$$
\max _{g_{n, 1}, g_{n, 2}} P_{1}\left(g_{1}^{G}-F d\right) g_{n, 1}-B g_{n, 1}+P_{2}\left(g_{2}^{G}+d\right) g_{n, 2}-B g_{n, 2}
$$

s.t. $g_{n, 1}, g_{n, 2} \geq 0$,

which has Karush-Kuhn-Tucker (KKT) conditions:

$$
\begin{aligned}
& 0 \leq-A_{1}+Z_{1} \cdot\left(g_{1}^{G}-F d\right)+Z_{1} g_{n, 1}+B \perp g_{n, 1} \geq 0 \\
& 0 \leq-A_{2}+Z_{2} \cdot\left(g_{2}^{G}+d\right)+Z_{2} g_{n, 2}+B \perp g_{n, 2} \geq 0 .
\end{aligned}
$$

We assume interior solutions to each firm's profit-maximisation problem (otherwise we have $g_{t}^{G}=0$ for at least one period, which is an uninteresting case). Combining this assumption with the KKT conditions and the known symmetry of the production levels gives:

$$
g_{1}^{G}(d)=\left(\frac{N}{N+1}\right)\left(\frac{A_{1}+Z_{1} F d-B}{Z_{1}}\right),
$$

and:

$$
g_{2}^{G}(d)=\left(\frac{N}{N+1}\right)\left(\frac{A_{2}-Z_{2} d-B}{Z_{2}}\right),
$$

as the aggregate production levels of the generators in the two periods. These production levels are given as functions of $d$, because of the impact that storage use has on the inverse demand functions in the two periods (and its resultant impact on generation levels).

Next, we substitute (1) and (2) into the periods-1 and -2 inverse demand functions, respectively, to obtain equilibrium prices in each period:

$$
p_{1}(d)=\frac{A_{1}+Z_{1} F d+B N}{N+1},
$$

and:

$$
p_{2}(d)=\frac{A_{2}-Z_{2} d+B N}{N+1}
$$

These two functions, $p_{1}(d)$ and $p_{2}(d)$, should be contrasted with the inverse demand functions, $P_{1}(x)$ and $P_{2}(x)$, which are defined in Section 2. The inverse demand functions represent what consumers are willing to pay in each of the two periods to consume $x \mathrm{MW}$. The two functions, $p_{1}(d)$ and $p_{2}(d)$, represent what the equilibrium market prices will be in each of the two periods if storage charges $F d$ MW in period 1 and discharges $d \mathrm{MW}$ in period 2. The functions, $p_{1}(d)$ and $p_{2}(d)$, give the equilibrium prices taking into account customers' willingness to pay via the inverse demand functions and the equilibrium production decisions of the generators, which are characterised by (1) and (2). 


\subsection{Investment and Operating Equilibrium of Profit-Maximising Storage Operator}

We analyse the behaviour of a profit-maximising storage operator by first examining its operating decision. Such a storage operator determines the amount of energy to charge and discharge in the two periods to maximise its profit. Thus, its operational problem is given by:

$$
\begin{array}{ll}
\max _{d} & d \cdot\left[p_{2}(d)-F p_{1}(d)\right] \\
\text { s.t. } & 0 \leq d \leq k,
\end{array}
$$

where $\mu$ is the Lagrange multiplier associated with the storage-capacity constraint. The KKT conditions for the storage-operation problem are:

$$
\begin{aligned}
& 0 \leq-\left(p_{2}(d)-F p_{1}(d)\right)-d p_{2}^{\prime}(d)+d F p_{1}^{\prime}(d)+\mu \perp d \geq 0 \\
& 0 \leq k-d \perp \mu \geq 0,
\end{aligned}
$$

where the derivatives of the price functions are taken with respect to $d$. Depending on the parameters, there are three possibilities for the solution, $d_{\Pi}^{*}(k)$ :

$$
d_{\Pi}^{*}(k)= \begin{cases}0, & \text { if } p_{2}(0)-F p_{1}(0)<0 ; \\ k, & \text { if } \frac{p_{2}(0)-F p_{1}(0)}{2\left[F p_{1}^{\prime}(0)-p_{2}^{\prime}(0)\right]} \geq k ; \\ \frac{p_{2}(0)-F p_{1}(0)}{2\left[F p_{1}^{\prime}(0)-p_{2}^{\prime}(0)\right]}, & \text { otherwise }\end{cases}
$$

where we write the operating decision as being a function of the investment level. This is to note explicitly that $d$ depends on $k$.

Equation (5) shows that for a fixed level of storage capacity there are three possible storage-use levels. The optimal choice among these three depends on the relative price difference between the on- and off-peak periods. The first case of no storage use only arises if the difference between on- and off-peak prices is too small for storing energy to be economic (i.e., if the price difference is too small to overcome the roundtrip efficiency losses). The third case in (5) is a level of storage use that equalises the marginal revenue that is earned from arbitraging the price difference between the on- and off-peak periods with the marginal cost that arises from diminishing the price difference between the two periods. This third case arises if the equalising storage-use level is less than the installed storage capacity. Otherwise, if the equalising storage-use level is greater than the installed storage capacity, the second case, in which storage is operated at its capacity, arises.

The three possible storage-use levels that are in (5) apply for a fixed storage-capacity level. Once we endogenise the storage-investment decision, which is the next step of our analysis, only the second case, in which all of the installed storage capacity is used, is optimal for the storage operator. This is because storage investment is costly (cf. Lemma 2). If the market is extended to include more than two operating periods, this property 'relaxes,' in the sense that the storage-capacity constraint is binding in at least one operating period. If it is not, the storage operator can slightly reduce its costly storage-investment level without impacting its ability to use storage at its desired level in any of the operating periods. 
We finally turn to the investment decision of the profit-maximising storage operator. Such a storage operator determines its investment level to maximise its profit from operating storage, less its investment cost. Thus, the investment problem is given by:

$$
\begin{aligned}
\max _{k} & {\left[p_{2}\left(d_{\Pi}^{*}(k)\right)-F p_{1}\left(d_{\Pi}^{*}(k)\right)\right] d_{\Pi}^{*}(k)-\left(I k^{2}\right) / 2 } \\
\text { s.t. } & k \geq 0 .
\end{aligned}
$$

Following the notation that is used in (5), we write the storage-operating decision as depending on the investment level. The period- 1 and -2 prices are written in the storage operator's objective function as depending on the operating-stage equilibrium. This is reflected by the prices' being defined as $p_{1}(\cdot)$ and $p_{2}(\cdot)$, which account for equilibrium production levels of the generating firms in characterising period-1 and -2 prices. Similarly, storage use is defined as $d_{\Pi}^{*}(k)$, which reflects profit-maximising storage use.

Before proceeding with analysing the storage-investment problem, we first show that the optimal storage-investment level is always equal to the amount of storage that is used in the operating stage.

Lemma 2 Consider a storage-investment problem of the form:

$$
\begin{array}{ll}
\max _{k, d} & \mathcal{P}(d)-\left(I k^{2}\right) / 2 \\
\text { s.t. } & 0 \leq d \leq k,
\end{array}
$$

where $\mathcal{P}(d)$ is an arbitrary function that directly depends only on the storage-use level, $d$, and $I>0$. Any optimal solution, $\left(k^{*}, d^{*}\right)$, must have $k^{*}=d^{*}$.

\section{Proof. See Appendix 7.1.}

From Lemma 2, we know that storage is fully utilised in the operating stage by the storage operator (i.e., that $d_{\Pi}^{*}(k)=k$ ). This is because our storage-investment problem has the form that is given in Lemma 2. Namely, storage investment only directly affects the objective function through its direct cost. Otherwise, the operating profit that the storage operator earns depends solely on the storage-use decision, $d$, and not on the storage-investment decision, $k$. That is to say, we can view the storage-investment problem as having the form in Lemma 2 with:

$$
\mathcal{P}(d)=\left[p_{2}(d)-F p_{1}(d)\right] d .
$$

Thus, based on Lemma 2, the storage-investment problem can be simplified to:

$$
\max _{k \geq 0}\left[p_{2}(k)-F p_{1}(k)\right] k-\left(I k^{2}\right) / 2
$$

The on- and off-peak energy prices that are in objective function (6) are written as functions of $k$ (as opposed to $d$ ) because storage is fully utilised in the operating stage. The simplified storage-investment problem is a convex quadratic program (due to the linear relationship between $p_{t}(k)$ and $k$ ). As such, the KKT condition, which is:

$$
0 \leq-p_{2}(k)+F p_{1}(k)+I k-k p_{2}^{\prime}(k)+F k p_{1}^{\prime}(k) \perp k \geq 0,
$$

is sufficient for a global optimum. We assume an interior solution of the storage-investment problem. Otherwise, we have $k=0$, which is an uninteresting case. Under this assumption, the KKT condition gives: 


$$
k_{\Pi}^{*}=\frac{A_{2}-F A_{1}-B N \cdot(F-1)}{I \cdot(N+1)+2 F^{2} Z_{1}+2 Z_{2}},
$$

so long as $A_{2}-F A_{1}-B N \cdot(F-1) \geq 0$, as the optimal investment level for the profit-maximising storage operator. We, hereafter, refer to $k_{\Pi}^{*}$ as the 'profit maximiser's storage-investment level.' The requirement that $A_{2}-F A_{1}-B N \cdot(F-1) \geq 0$ intuitively means that the price difference between the on- and off-peak periods has to be sufficiently high to justify the cost of storage investment. If this condition is not satisfied, then we have the uninteresting case in which zero storage investment is optimal.

\subsection{Investment and Operating Equilibrium of Welfare-Maximising Storage Operator}

We analyse the case of a welfare-maximising storage operator in the same way that we do a profit maximiser. That is, we first examine its operating decisions and then determine its investment level. With a welfare-maximising storage operator, the operating decisions of generators are still given by (1) and (2). To determine the operating and investment decisions of a welfare-maximising storage operator, we first characterise the components of social welfare. Period- $t$ consumer welfare is defined as the integral of the difference between customers' willingness to pay for energy, which is given by $P_{t}(x)$, and the equilibrium energy price, which is given by $p_{t}(d)$. Thus, periods- 1 and -2 consumer welfare, as a function of $d$, are:

$$
W_{1}^{C}(d)=\int_{0}^{g_{1}^{G}(d)-F d}\left[P_{1}(x)-p_{1}(d)\right] d x=\frac{1}{2} Z_{1} \cdot\left[g_{1}^{G}(d)-F d\right]^{2},
$$

and:

$$
W_{2}^{C}(d)=\int_{0}^{g_{2}^{G}(d)+d}\left[P_{2}(x)-p_{2}(d)\right] d x=\frac{1}{2} Z_{2} \cdot\left[g_{2}^{G}(d)+d\right]^{2}
$$

respectively. Periods-1 and -2 producer welfare are:

$$
W_{1}^{G}(d)=g_{1}^{G}(d) \cdot\left[p_{1}(d)-B\right]=\frac{Z_{1}}{N} g_{1}^{G}(d)^{2},
$$

and:

$$
W_{2}^{G}(d)=g_{2}^{G}(d) \cdot\left[p_{2}(d)-B\right]=\frac{Z_{2}}{N} g_{2}^{G}(d)^{2}
$$

respectively. The welfare of the storage operator is given by:

$$
\begin{aligned}
W^{S}(d, k) & =d \cdot\left[p_{2}(d)-F p_{1}(d)\right]-\frac{1}{2} I k^{2} \\
& =\frac{d}{N+1}\left\{k_{\Pi}^{*}\left[I \cdot(N+1)+2 F^{2} Z_{1}+2 Z_{2}\right]-d Z_{2}-d F^{2} Z_{1}\right\}-\frac{1}{2} I k^{2} .
\end{aligned}
$$

The storage operator's welfare is given by two terms. The first, $d \cdot\left[p_{2}(d)-F p_{1}(d)\right]$, represents the intertemporal-arbitrage profit earned, which depends on the operating-stage decision, $d$. The second, $\left(I k^{2}\right) / 2$, represents the storage operator's investment cost, which depends on $k$.

Using these expressions for the various components of social welfare, the operating problem for the welfare-maximising storage operator is: 


$$
\begin{gathered}
\max _{d}\left[W_{1}^{C}(d)-W_{1}^{C}(0)\right]+\left[W_{2}^{C}(d)-W_{2}^{C}(0)\right]+\left[W_{1}^{G}(d)-W_{1}^{G}(0)\right]+\left[W_{2}^{G}(d)-W_{2}^{G}(0)\right] \\
+\left[p_{2}(d)-F p_{1}(d)\right] d
\end{gathered}
$$

s.t. $0 \leq d \leq k$,

where $\mu$ is the Lagrange multiplier associated with the upper bound on $d$. The first four bracketed terms in (8) represent the consumer and producer welfare in the two operating periods. The last term in (8) represents the operating profit of the storage plant only (i.e., storage-investment cost is not accounted for). The reason that storage-investment cost is not included in (8) is that the investment cost is sunk in the operating stage, and, as such, does not affect the social welfare engendered by storage use.

Next, using the expressions for $g_{1}^{G}(d)$ and $g_{2}^{G}(d)$, we have:

$$
\begin{aligned}
& W_{1}^{C^{\prime}}(d)=-F \frac{Z_{1}}{(N+1)^{2}}\left[N \frac{A_{1}-B}{Z_{1}}-F d\right], \\
& W_{2}^{C^{\prime}}(d)=\frac{Z_{2}}{(N+1)^{2}}\left[N \frac{A_{2}-B}{Z_{2}}+d\right], \\
& W_{1}^{G^{\prime}}(d)=F \frac{2 Z_{1} N}{(N+1)^{2}}\left[\frac{A_{1}-B}{Z_{1}}+F d\right], \\
& W_{2}^{G^{\prime}}(d)=-\frac{2 Z_{2} N}{(N+1)^{2}}\left[\frac{A_{2}-B}{Z_{2}}-d\right],
\end{aligned}
$$

and:

$$
\frac{\partial}{\partial d} W^{S}(d, k)=\left(k_{\Pi}^{*}-d\right) \frac{2 F^{2} Z_{1}+2 Z_{2}}{N+1}+k_{\Pi}^{*} I .
$$

Taken together, these give us:

$$
\begin{array}{r}
\frac{\partial}{\partial d} W(d, k)=F \frac{Z_{1}}{(N+1)^{2}}\left[N \frac{A_{1}-B}{Z_{1}}+(2 N+1) F d\right]+\frac{Z_{2}}{(N+1)^{2}}\left[(2 N+1) d-N \frac{A_{2}-B}{Z_{2}}\right] \\
+\left(k_{\Pi}^{*}-d\right) \frac{2 F^{2} Z_{1}+2 Z_{2}}{N+1}+k_{\Pi}^{*} I,
\end{array}
$$

as the derivative of social welfare with respect to $d$. We also have that:

$$
\begin{aligned}
\frac{\partial}{\partial d} W(0, k) & =F N \frac{A_{1}-B}{(N+1)^{2}}-N \frac{A_{2}-B}{(N+1)^{2}}+\frac{A_{2}-F A_{1}-B N \cdot(F-1)}{N+1} \\
& =\frac{A_{2}-F A_{1}+B \cdot(N+1)^{2}(1-F)-B \cdot(1-F)}{(N+1)^{2}} .
\end{aligned}
$$

Using these derivatives, the KKT conditions for the welfare maximiser's operational problem, which is given by (8) and (9), are: 


$$
\begin{gathered}
0 \leq \frac{F Z_{1}}{(N+1)^{2}}\left[\frac{N\left(A_{1}-B\right)}{Z_{1}}-F d\right]-\frac{Z_{2}}{(N+1)^{2}}\left[\frac{N\left(A_{2}-B\right)}{Z_{2}}+d\right]-\frac{2 F Z_{1} N}{(N+1)^{2}}\left[\frac{\left(A_{1}-B\right)}{Z_{1}}+F d\right] \\
+\frac{2 Z_{2} N}{(N+1)^{2}}\left[\frac{\left(A_{2}-B\right)}{Z_{1}}-d\right]-\frac{\left(A_{2}-Z_{2} d+B N\right)}{N+1}+F \frac{\left(A_{1}-Z_{1} F d+B N\right)}{N+1} \\
-\left(p_{2}(d)-F p_{1}(d)\right)-d p_{2}^{\prime}(d)+d F p_{1}^{\prime}(d)+\mu \perp d \geq 0 \\
0 \leq k-d \perp \mu \geq 0 .
\end{gathered}
$$

Depending on the parameters, there are three possible solutions, $d_{W}^{*}(k)$ :

$$
d_{W}^{*}(k)= \begin{cases}0, & \text { if } A_{2}-F A_{1}-B N \cdot(F-1)(N+2)<0 \\ k, & \text { if } \frac{A_{2}-F A_{1}-B N \cdot(F-1)(N+2)}{F^{2} Z_{1}+Z_{2}} \geq k ; \\ \frac{A_{2}-F A_{1}-B N \cdot(F-1)(N+2)}{F^{2} Z_{1}+Z_{2}}, & \text { otherwise. }\end{cases}
$$

The three storage-use levels that are in (11) have analogous interpretations to those that are in (5), which corresponds to a profit-maximising storage operator. Namely, the first case in (11) arises if the welfare (as opposed to profit) gains from storage use are insufficient to justify the roundtrip efficiency losses that arise from storage use. In such a case, storage is not used. The third case uses storage at a level that the marginal net welfare gains are exactly zero (meaning that there are no benefits from increasing or decreasing storage use), so long as this storage-use level is below the installed capacity. If this level of storage use that achieves zero marginal net welfare gains is greater than $k$, the second case, in which storage is operated at its capacity, arises. As with a profit-maximising storage operator, the three storage-use levels that are in (11) can only arise with an exogenously fixed storage-investment level. Once the storage-investment decision is endogenised, Lemma 2 gives us the same result as with a profit-maximising storage operator that the optimal storage-investment level is exactly equal to the amount of storage that is used in the operating stage.

We finally turn to the welfare-maximiser's investment decision. The welfare-maximising storage operator determines its investment level to maximise social welfare (including the storage-investment cost, which is not sunk at the investment stage). The investment problem is given by:

$$
\begin{aligned}
\max _{k \geq 0}[ & \left.W_{1}^{C}\left(d_{W}^{*}(k)\right)-W_{1}^{C}(0)\right]+\left[W_{2}^{C}\left(d_{W}^{*}(k)\right)-W_{2}^{C}(0)\right]+\left[W_{1}^{G}\left(d_{W}^{*}(k)\right)-W_{1}^{G}(0)\right] \\
+ & {\left[W_{2}^{G}\left(d_{W}^{*}(k)\right)-W_{2}^{G}(0)\right]+\left[p_{2}\left(d_{W}^{*}(k)\right)-F p_{1}\left(d_{W}^{*}(k)\right)\right] d_{W}^{*}(k)-\frac{1}{2} I k^{2} . }
\end{aligned}
$$

This problem follows the notational conventions that are used in formulating the investment problem of the profit-maximising storage operator. The period- 1 and -2 prices are defined as $p_{1}(\cdot)$ and $p_{2}(\cdot)$, which account for the equilibrium production levels of the generating firms. Storage use is defined as $d_{W}^{*}(k)$, which characterises its use by the welfare-maximising storage operator.

By appealing to Lemma 2, we know that storage is fully utilised in the operating stage (i.e., that $\left.d_{W}^{*}(k)=k\right)$. We further assume an interior solution to the storage-investment problem (i.e., that $k>0$ ). Otherwise, we have an uninteresting case with no storage investment. Under these assumptions, the storage-investment problem simplifies to the convex quadratic program: 


$$
\begin{gathered}
\max _{k}\left[W_{1}^{C}(k)-W_{1}^{C}(0)\right]+\left[W_{2}^{C}(k)-W_{2}^{C}(0)\right]+\left[W_{1}^{G}(k)-W_{1}^{G}(0)\right] \\
+\left[W_{2}^{G}(k)-W_{2}^{G}(0)\right]+\left[p_{2}(k)-F p_{1}(k)\right] k-\frac{1}{2} I k^{2} .
\end{gathered}
$$

As in the case of a profit-maximising storage operator, the on- and off-peak energy prices are written as functions of $k$ (as opposed to $d$ ) because of the assumption that storage is fully utilised. The necessary and sufficient KKT condition is:

$$
\begin{aligned}
\left\{F^{2} \frac{Z_{1}(2 N+1)}{N+1}+\frac{Z_{2}(2 N+1)}{N+1}-\left[I \cdot(N+1)+2 F^{2} Z_{1}+2 Z_{2}\right]\right\} k \\
=\frac{N}{N+1}\left[A_{2}-B-F \cdot\left(A_{1}-B\right)\right]-k_{\Pi}^{*}\left[I \cdot(N+1)+2 F^{2} Z_{1}+2 Z_{2}\right],
\end{aligned}
$$

which gives:

$$
k_{W}^{*}=\frac{A_{2}-F A_{1}-B N \cdot(F-1)(N+2)}{I \cdot(N+1)^{2}+F^{2} Z_{1}+Z_{2}},
$$

as the investment level of the welfare-maximising storage operator, so long as $A_{2}-F A_{1}-B N$. $(F-1)(N+2) \geq 0$. We, hereafter, refer to $k_{W}^{*}$ as the 'welfare maximiser's storage-investment level.' The requirement that $A_{2}-F A_{1}-B N \cdot(F-1)(N+2) \geq 0$ means that the price difference between the on- and off-peak periods has to be sufficiently high to provide enough welfare gains from storage use to justify its investment cost. If this condition is not satisfied, then we have the uninteresting case of no storage investment.

\subsection{Welfare Analysis}

We conclude our analysis of the case with constant marginal generation costs by examining the welfare implications of having a profit- or welfare-maximising storage operator. We begin with the following proposition, which gives conditions under which the storage-investment levels of the two types of storage operators differ. The proposition also shows that infinitesimal changes in the profit maximiser's storage-investment level toward the welfare maximiser's level are always welfare-enhancing.

Proposition 1 The welfare maximiser's storage-investment level is larger (smaller) than the profit maximiser's storage-investment level if the number of generating firms is less (greater) than $\bar{N}$, which is the unique positive root of the characteristic quadratic:

$$
\begin{aligned}
Q(N)= & -N^{2} \cdot\left[I \cdot\left(A_{2}-F A_{1}\right)+I B \cdot(F-1)+2\left(F^{2} Z_{1}+Z_{2}\right) B \cdot(F-1)\right] \\
& -N \cdot\left[I \cdot\left(A_{2}-F A_{1}\right)+I B \cdot(F-1)+3\left(F^{2} Z_{1}+Z_{2}\right) B \cdot(F-1)\right]+\left(A_{2}-F A_{1}\right)\left(F^{2} Z_{1}+Z_{2}\right) .
\end{aligned}
$$

Moreover, starting from the profit maximiser's storage-investment level, an infinitesimal increase (decrease) in storage capacity is always welfare-enhancing for $N<\bar{N}(N>\bar{N})$.

\section{Proof. See Appendix 7.1.}

Proposition 1 shows that the welfare maximiser's storage-investment level is greater than the profit maximiser's storage-investment level only if the number of generating firms is relatively small. Intuitively, with a small number of generators, the ability of the generating firms to exercise 
market power (especially during the on-peak period) results in high deadweight losses. As such, adding storage increases social welfare by allowing greater supply during the on-peak period. Thus, the welfare-maximising storage operator invests in more storage than a profit maximiser does for a small number of firms. The situation is reversed for a large number of generators. The second part of the proposition shows that infinitesimal changes to the profit maximiser's storage-investment level are always welfare-enhancing. Whether additions or subtractions of storage capacity are welfare-enhancing depends on whether the profit maximiser invests in more or less storage capacity than the welfare maximiser does (i.e., whether the number of firms is less or greater than $\bar{N}$ ).

The following proposition demonstrates that the threshold number of firms, $\bar{N}$, decreases with both the investment- and generation-cost parameters. That is to say, the welfare maximiser's storage-investment level being higher than the profit maximiser's storage-investment level is less likely as the marginal cost of either storage investment or generation increase.

Proposition $2 \quad \bar{N}$ is strictly decreasing in the storage-investment-cost parameter, I, and is strictly decreasing in the generation-cost parameter, $B$.

Proof. See Appendix 7.1.

The following proposition examines the impacts of the market structure on the price differential between the on- and peak periods. It shows that greater storage use or a greater number of generating firms decrease the price difference.

Proposition 3 The equilibrium price differential, $p_{2}(d)-F p_{1}(d)$, decreases with storage use. It also decreases with more generating firms.

Proof. See Appendix 7.1.

Intuitively, the number of generating firms affects the price differential because the exercise of market power increases off- and on-peak equilibrium prices. However, the effect is more pronounced during the on-peak period. Thus, fewer generating firms increases the price differential. Conversely, the reduction of market power, viz., by having more firms, decreases the price differential.

Building on this result, the following proposition shows that both the welfare and profit maximiser's storage-investment levels decrease with the number of generating firms. Intuitively, having more generating firms makes the generation equilibrium more competitive, which reduces the price differential between on- and off-peak periods. As such, a profit-maximising storage operator has less incentive to invest in storage, because there is a smaller price difference to arbitrage. A welfare-maximising storage operator has less incentive to invest in storage with more firms, because the more competitive generation equilibrium implies that there are less welfare gains from the use of storage.

Proposition 4 The profit maximiser's and welfare maximiser's storage-investment levels decrease with more generating firms.

Proof. See Appendix 7.1.

Finally, the following proposition and corollary show that there are instances, with a relatively competitive generation sector, in which the profit maximiser's storage-investment level results in lower social welfare than having no storage at all. This result challenges the conventional wisdom that energy storage is always welfare enhancing. Intuitively, the result arises because 'too much' storage is adopted by the profit-maximising storage operator when $N$ is greater than a critical 
threshold, $\tilde{N}$. The profit maximiser adopts too much storage in such cases because the highly competitive generation sector results in relatively small price differentials between the on- and off-peak periods. As such, the storage operator invests in more capacity to store (and sell) a greater volume of energy to partially overcome the smaller price margin.

Proposition 5 The total social welfare that is achieved by the profit maximiser's storage-investment level, $W\left(k_{\Pi}^{*}\right)$, is strictly greater (less) than the social welfare that is achieved without any storage investment, $W(0)$, so long as the number of firms is less (greater) than some critical threshold, $\tilde{N}$.

Proof. See Appendix 7.1.

Corollary 1 If the social welfare that is engendered by the profit maximiser's storage-investment level, $W\left(k_{\Pi}^{*}\right)$, is strictly less than the social welfare that is obtained without storage, $W(0)$, then we have that $\bar{N}<\tilde{N}$.

Proof. See Appendix 7.1.

Proposition 5 should be contrasted with the welfare analysis that is conducted by Sioshansi (2014). Sioshansi (2014) shows that if the amount of storage is exogenously fixed, there can be no welfare losses arising from storage use with a perfectly competitive generation sector. Proposition 5 , conversely, shows that if the storage-investment decision is endogenised, there are guaranteed to be welfare losses arising from storage investment and use by a profit maximiser, so long as the generation sector is sufficiently competitive. This shows the sensitivity of the welfare impacts of energy storage to endogenously capturing the investment decision.

\section{INCENTIVISATION OF STORAGE INVESTMENT WITH A RAMPING CHARGE}

Our analysis in Section 3 demonstrates that the profit and welfare maximisers' storage-investment levels typically differ. One instrument for driving the profit maximiser toward a more economically efficient level of storage investment is through a ramping charge on generation. To understand the premise of this mechanism, for a low (high) number of generating firms, 'too little' ('too much') storage capacity is installed by the profit maximiser. In other words, the price differential between the on- and off-peak periods is 'too high' ('too low') for a relatively small (large) number of generators. Consequently, with a profit-maximising storage operator, generators are ramping their production more (less) than occurs with a welfare maximiser.

Hence, we examine the impacts of imposing an exogenous ramping charge, which we denote $R$ and which is measured in $\$ / \mathrm{MW}$, on generation. This ramping charge can take on a negative value, representing a ramping payment to generators. The concept of a ramping charge is implicitly used in many wholesale electricity markets today. Zhao et al. (2017) demonstrate that if ramping constraints are binding in a unit commitment- or economic dispatch-based market model that represents such constraints, the resulting Lagrange multipliers implicitly incorporate the cost of the ramping constraints in determining market-clearing prices. Thus, our proposed solution builds off of market-design principles that are in-use today.

To analyse the impact of the ramping charge, we again begin by first examining the production decisions of the generators. Generator $n$ 's profit-maximisation problem is given by:

$$
\max _{g_{n, 1}, g_{n, 2} \geq 0} P_{1}\left(g_{1}^{G}-F d\right) g_{n, 1}-B g_{n, 1}+P_{2}\left(g_{2}^{G}+d\right) g_{n, 2}-B g_{n, 2}-R \cdot\left(g_{n, 2}-g_{n, 1}\right) \text {. }
$$


Note that this problem can be written as:

$$
\max _{g_{n, 1}, g_{n, 2} \geq 0} P_{1}\left(g_{1}^{G}-F d\right) g_{n, 1}-(B-R) g_{n, 1}+P_{2}\left(g_{2}^{G}+d\right) g_{n, 2}-(B+R) g_{n, 2},
$$

meaning that the ramping charge can be viewed as changing the (still symmetric) marginal production costs of the generators in the two periods. As such, we can appeal to Lemma 1 to conclude that with the ramping charge the production equilibrium is still symmetric. Assuming an interior solution to the generators' profit-maximisation problems, we have aggregate production levels:

$$
\hat{g}_{1}^{G}(d, R)=\left(\frac{N}{N+1}\right)\left(\frac{A_{1}+Z_{1} F d-B+R}{Z_{1}}\right),
$$

and:

$$
\hat{g}_{2}^{G}(d, R)=\left(\frac{N}{N+1}\right)\left(\frac{A_{2}-Z_{2} d-B-R}{Z_{2}}\right),
$$

in the two periods. We write these production levels as depending on both $d$ and $R$, to explicitly note the effect of the ramping charge. Comparing these two expressions with (1) and (2), respectively, shows that the ramping charge has the desired impact on generators' production decisions. Namely, a positive value of $R$ disincentivises having a large difference between on- and off-peak production levels. As such, $R$ increases off-peak production while contemporaneously decreasing on-peak production. A negative value of $R$ has the reversed effect on the two production levels.

Substituting (13) and (14) into the period-1 and -2 inverse demand functions, respectively, gives equilibrium prices:

$$
\hat{p}_{1}(d, R)=\frac{A_{1}+Z_{1} F d+N \cdot(B-R)}{N+1}
$$

and:

$$
\hat{p}_{2}(d, R)=\frac{A_{2}-Z_{2} d+N \cdot(B+R)}{N+1},
$$

in the two periods. Comparing these price functions to (3) and (4), respectively, shows the impact of the changed production levels (that are caused by the ramping charge) on off- and on-peak energy prices. As noted before, a positive value of $R$ yields greater off-peak and reduced on-peak production. This, in turn, results in lower off-peak and higher on-peak prices (in order for demand to equal supply). This higher price differential between the on- and off-peak periods gives greater incentives for storage investment. A negative value of $R$ has the opposite effect. the problem:

We next model the operating decisions of the profit-maximising storage operator as solving

$$
\max _{d \in[0, k]} d \cdot\left[\hat{p}_{2}(d, R)-F \hat{p}_{1}(d, R)\right],
$$

which yields the optimal solution:

$$
\hat{d}_{\Pi}(k, R)= \begin{cases}0, & \text { if } \hat{p}_{2}(0, R)-F \hat{p}_{1}(0, R)<0 ; \\ k, & \text { if } \frac{\hat{p}_{2}(0, R)-F \hat{p}_{1}(0, R)}{2\left[F \hat{p}_{1}^{\prime}(0, R)-\hat{p}_{2}^{\prime}(0, R)\right]} \geq k ; \\ \frac{\hat{p}_{2}(0, R)-F \hat{p}_{1}(0, R)}{2\left[F \hat{p}_{1}^{\prime}(0, R)-\hat{p}_{2}^{\prime}(0, R)\right]}, & \text { otherwise. }\end{cases}
$$


The three storage-use levels that are given in this equation have analogous interpretations to those that are in (5), but taking account of the effect of the ramping charge on the prices in the two periods. As in the case of the profit-maximising storage operator in the absence of a ramping charge, only the second case, in which all of the installed storage capacity is used, arises once the storage-investment decision is endogenised.

We finally have the profit maximiser's storage-investment problem:

$$
\max _{k \geq 0}\left[\hat{p}_{2}\left(\hat{d}_{\Pi}(k, R), R\right)-F \hat{p}_{1}\left(\hat{d}_{\Pi}(k, R), R\right)\right] \hat{d}_{\Pi}(k, R)-\left(I k^{2}\right) / 2 .
$$

From Lemma 2, we know that $\hat{d}_{\Pi}(k, R)=k$. We further assume that $k>0$, which would otherwise yield an uninteresting case, which gives:

$$
\hat{k}_{\Pi}(R)=\frac{A_{2}-F A_{1}-B N \cdot(F-1)+R N \cdot(F+1)}{I \cdot(N+1)+2 F^{2} Z_{1}+2 Z_{2}},
$$

as the optimal storage-investment level of the profit maximiser, so long as $A_{2}-F A_{1}-B N$. $(F-1)+R N \cdot(F+1) \geq 0$. As stated above, a positive ramping charge increases the price differential between the on- and off-peak periods. This increased price difference incentivises greater storage investment by the profit-maximising storage operator. This impact of the ramping charge is conveyed through the final term in the numerator of (15).

We can finally determine the value for the ramping charge that results in the profit and welfare maximisers' storage-investment levels being equal to one another by solving $\hat{k}_{\Pi}(R)=k_{W}^{*}$, which gives:

$$
R^{*}=\frac{\left(A_{2}-F A_{1}\right)[\zeta-I N \cdot(N+1)]-B N \cdot(F-1)[I \cdot(N+1)+(2 N+3) \zeta]}{N \cdot(F+1)\left[I \cdot(N+1)^{2}+F^{2} Z_{1}+Z_{2}\right]},
$$

where $\zeta=F^{2} Z_{1}+Z_{2}$. We, hereafter, refer to $R^{*}$ as the 'equalising ramping charge.' An interesting property of a ramping charge, and the equalising ramping charge in particular, is that it may induce greater or less social welfare than that achieved by the welfare-maximising storage operator in the absence of a ramping charge. This is because the ramping charge provides an added layer of control that can both affect the level of storage investment and the production decisions of the generating firms. As such, it could be used to correct productive-efficiency losses from the exercise of market power by the generating firms as well as suboptimal storage-investment and -use decisions made by a profit-maximising storage operator.

In fact, one could optimise the ramping charge in a tri-level model. In such an analysis the upper-most level represents a welfare-maximising social planner, which chooses $R$. The middle-level problem represents the investment decision of the storage operator (which could be profitor welfare-maximising) while the lower-level problem gives the equilibrium between generators and the storage operator in the two operating periods. If such an exercise is conducted, it should be noted that the ramping charge that we examine is one of many possible approaches to address any welfare losses that arise from the exercise of market power by a storage operator or the generators. Market monitoring, forced divestiture, or incentivising entry are among other means of addressing market failures. As such, a ramping charge is at most a heuristic approach to addressing market inefficiencies. We do not conduct such a tri-level modelling exercise. Rather, we simply find a value of $R$ that modifies the amount of storage that is built. As such, we may arrive at equilibria that either increase or decrease social welfare compared to the equilibrium that is attained with a welfare-maximising storage operator. The difference in social welfare between the case of a profit-maximising 
storage operator with a ramping charge and the case of a welfare-maximising storage operator without one is given by:

$$
W\left(\hat{k}_{\Pi}(R)\right)-W\left(k_{W}^{*}\right)=-\frac{N^{2} R^{2}}{2(N+1)^{2}}\left(\frac{1}{Z_{1}}+\frac{1}{Z_{2}}\right)+\frac{R N}{(N+1)^{2}}\left(\frac{A_{1}+F Z_{1} k_{W}^{*}-B}{Z_{1}}-\frac{A_{2}-Z_{2} k_{W}^{*}-B}{Z_{2}}\right) .
$$

As a benchmark, and assuming that all installed storage capacity is fully utilised, we can examine the problem of a hypothetical social planner that controls all generation and storage decisions without the need for ramping charges:

$$
\max _{g_{1}, g_{2}, k \geq 0} A_{1} \cdot\left(g_{1}-F k\right)-\frac{Z_{1}}{2}\left(g_{1}-F k\right)^{2}+A_{2} \cdot\left(g_{2}+k\right)-\frac{Z_{2}}{2}\left(g_{2}+k\right)^{2}-B \cdot\left(g_{1}+g_{2}\right)-\frac{1}{2} I k^{2} .
$$

This social planner's problem has the optimal solution:

$$
\begin{aligned}
& g_{1}^{P}=\frac{A_{1}-B}{Z_{1}}, \\
& g_{2}^{P}=\frac{A_{2}-B}{Z_{2}},
\end{aligned}
$$

and:

$$
k^{P}=0 .
$$

Indeed, neither storage nor ramping charges are needed in this ideal situation, because it is possible for the social planner to control generation exactly to match the marginal benefit from consumption to marginal cost in each time period. Hence, unlike the central-planning result with a linear marginal cost (cf. Appendix 7.2), any storage use or investment is non-optimal with a constant marginal generation cost under centralised planning. Because $k^{P}=0$ is the optimal amount of storage in this central-planning benchmark, we define the notation, $W\left(g_{1}^{P}, g_{2}^{P}, k^{P}\right)$, to give the total social welfare in the central-planning case.

\section{NUMERICAL EXAMPLES}

We present the results of two sets of numerical examples here. First, we summarise results for an example with constant marginal generation costs, which is the case that is examined in Sections 3 and 4. Then we provide numerical results for a case in which the marginal generation costs are linear. Table 1 summarises the parameter values that are used in our numerical case studies. The parameter, $K$, is related to the slope of the generators' marginal cost for the cases that are analysed in Section 5.2 and Appendix 7.2, in which the generation firms are assumed to have linear marginal costs. We allow $N$ to range between 1 and 10 , to show the impacts of different levels of market competitiveness on storage-investment decisions.

\subsection{Results with Constant Marginal Generation Costs}

Figure 1 illustrates the characteristic polynomial (cf. Proposition 1) for the parameter values that are given in Table 1 and for two other sets of values for $I$ and $B$. For the base parameter values that are given in Table 1, the two roots of the characteristic polynomial are -2.577 and 1.555 (cf. the solid blue curve in Figure 1), meaning that with a single generating firm the profit maximiser's 
Table 1: Data for Numerical Examples

\begin{tabular}{llc}
\hline Parameter & Definition & Value \\
\hline$A_{1}$ & Intercept of off-peak inverse demand function & 200 \\
$A_{2}$ & Intercept of on-peak inverse demand function & 400 \\
$Z_{1}$ & Slope of off-peak inverse demand function & 10 \\
$Z_{2}$ & Slope of on-peak inverse demand function & 10 \\
$B$ & Marginal generation cost & 20 \\
$K$ & Slope coefficient of marginal generation cost (for linear-marginal cost) & 1 \\
$I$ & Investment-cost coefficient of energy storage & 5 \\
$E$ & Roundtrip efficiency of energy storage & 0.95 \\
\hline
\end{tabular}

Figure 1: Characteristic polynomial, $Q(N)$, for different values of $I$ and $B$ with constant marginal generation costs.

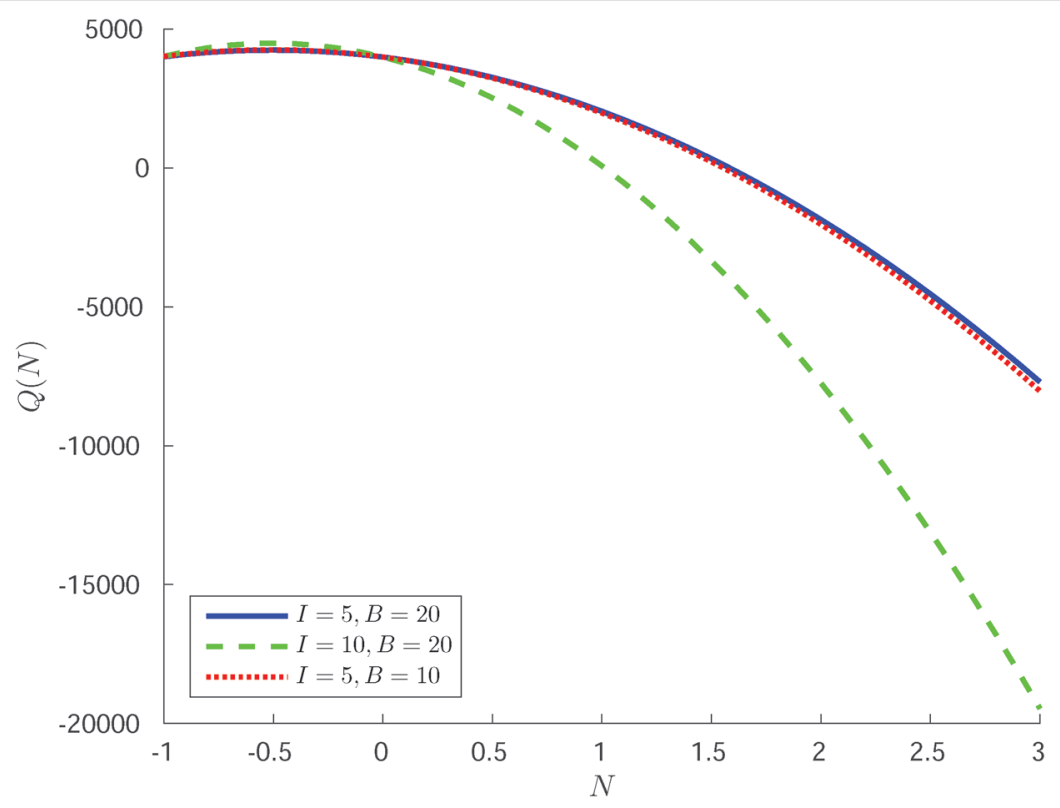

storage-investment level is less than the welfare maximiser's storage-investment level. Otherwise, with two or more generating firms, the profit maximiser's storage-investment level is higher.

The two other curves in Figure 1 illustrate the results of Proposition 2. First, the dashed green curve shows that if the storage-investment cost increases, the threshold value, $\bar{N}$, decreases. The red dotted curve shows that decreasing the marginal generation cost has the reversed effect on $\bar{N}$. Figure 2 further illustrates Proposition 1 by showing the profit and welfare maximisers' storage-investment levels, assuming the base parameter values that are given in Table 1. It shows that with more than two generating firms the profit-maximising storage operator over-invests relative to the welfare maximiser. Figure 2 also illustrates Proposition 4, in that the profit and welfare maximisers' storage-investment levels are decreasing in the number of firms.

Figure 3 shows equilibrium on- and off-peak prices with different numbers of generating firms and with different storage-investment decisions. As expected, a case with no storage yields the greatest price differences. This is because there is no opportunity to substitute low-price electricity that is generated off-peak for high-price on-peak electricity. The equilibrium price differentials with profit-maximising and welfare-maximising storage operators vary, but follow the intuition behind Proposition 1. If $N \leq \bar{N}$, Proposition 1 tells us that the welfare-maximising storage operator invests 
Figure 2: Profit and welfare maximisers' storage-investment levels with constant marginal generation costs.

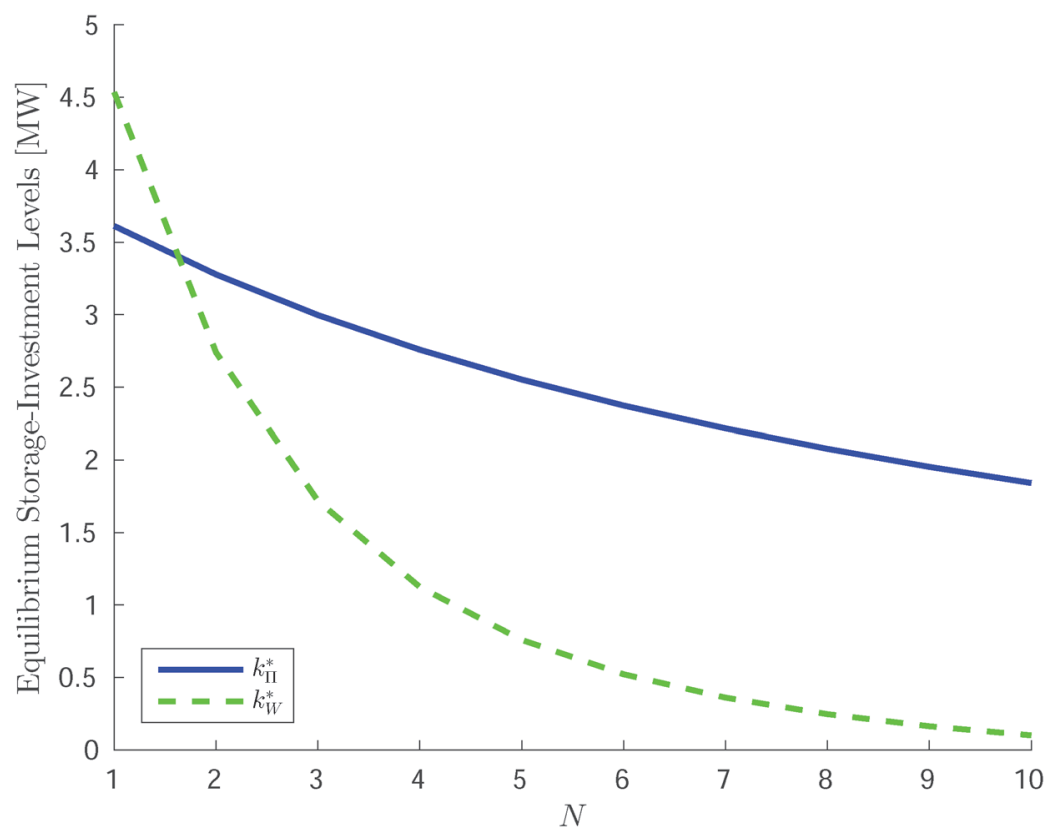

Figure 3: Energy prices under different storage-investment equilibria with constant marginal generation costs.

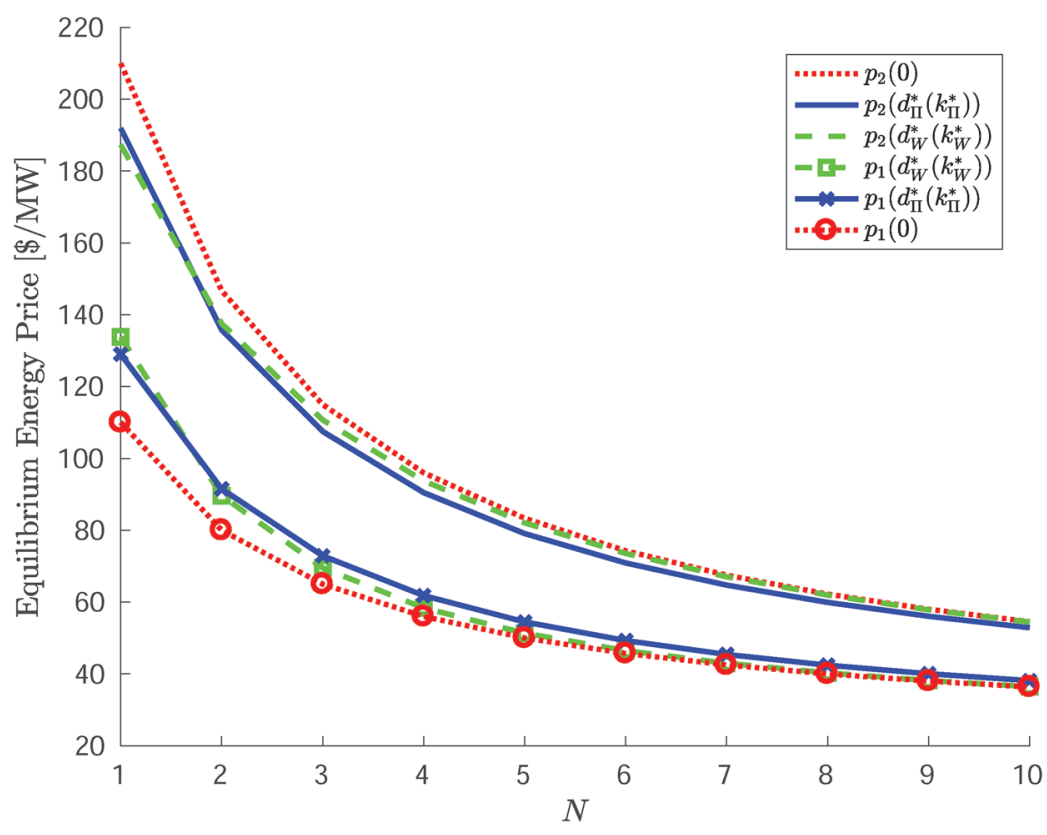

in more storage capacity than the profit maximiser does. This is because with few generating firms there are relatively large deadweight losses from their exercise of market power. We know from Lemma 2 that all of the storage is utilised. This implies that the price differential will be smaller with a welfare-maximising storage operator compared to a profit maximiser. If there are more than 


\section{Figure 4: Change in social welfare relative to no-storage case under different storage- investment equilibria with constant marginal generation costs.}

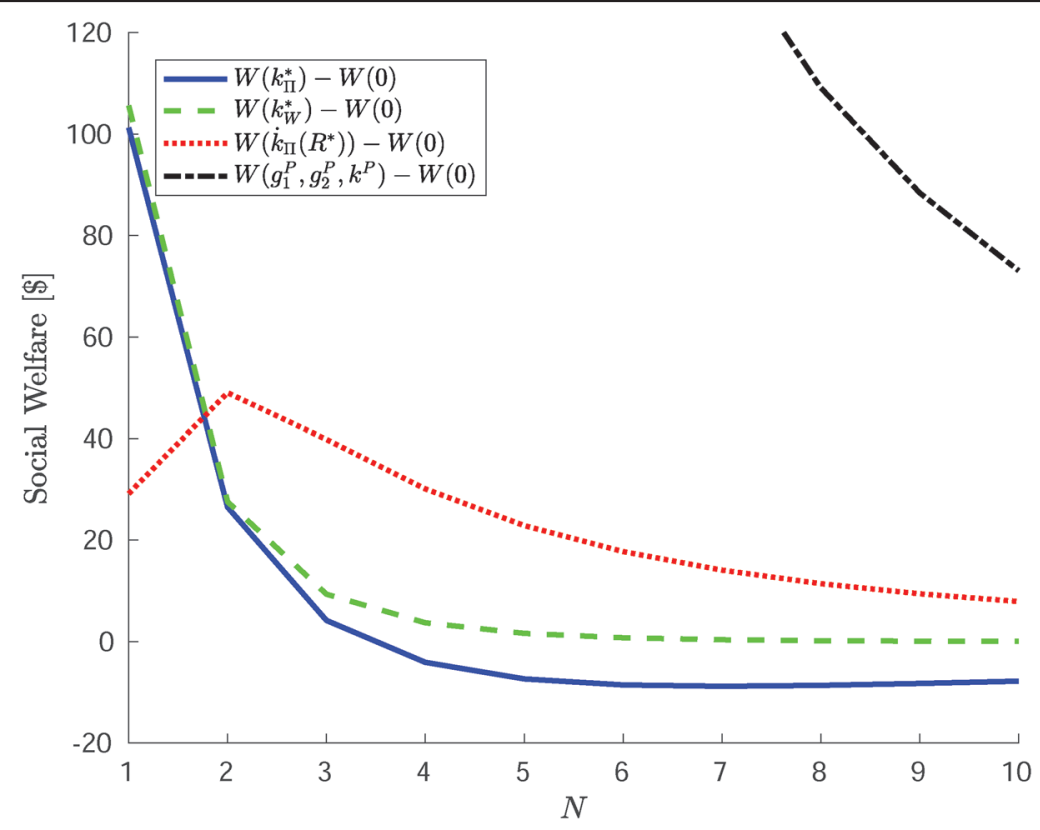

$\bar{N}$ generating firms, Proposition 1 implies that the relative investment levels are reversed, meaning that there is a smaller price differential with a profit-maximising storage operator.

Figure 4 shows the change in social welfare that is engendered by the profit and welfare maximisers' storage-investment levels relative to the no-storage case. It also shows the change in social welfare from the equilibrium that arises with a profit-maximising storage operator in the presence of a ramping charge that is set at $R^{*}$, the equalising ramping charge. The figure finally shows the change in social welfare under centralised planning.

The figure demonstrates that the social welfare that is engendered by the profit maximiser's storage-investment level is always lower than that arising from the welfare maximiser's storage-investment level, which is keeping with Proposition 1. The figure also shows that the profit maximiser's storage-investment level can result in lower social welfare compared to having no storage at all. There are social welfare losses arising from the profit maximiser's storage-investment level with four or more generating firms. This is keeping with Proposition 5. Indeed, from the proof of Proposition 5 we can compute the threshold number of firms, $\tilde{N}=3.389$, above which the social welfare that is achieved with the profit maximiser's storage-investment level is less than the no-storage case.

Figure 4 also shows that the social welfare that arises from the welfare maximiser's storage-investment level asymptotes to the social welfare in the no-storage case as $N \rightarrow+\infty$ (i.e., the social-welfare difference approaches zero). This is because as $N \rightarrow+\infty$ the generation sector becomes increasingly competitive, meaning that the off- and on-peak prices approach the marginal generation cost, $B$. As that happens, storage becomes less necessary for mitigating the exercise of market power. Indeed, as discussed at the end of Section 4 and shown in (16), if the generation sector is perfectly competitive (which is functionally equivalent to the assumption of centralised planning), zero storage investment is welfare-maximising.

Figure 4 also illustrates the welfare effects of the ramping charge. Proposition 1 does not hold with the ramping charge (i.e., the welfare impact of increasing storage capacity from the level 
Figure 5: Equalising ramping charge with constant marginal generation costs.

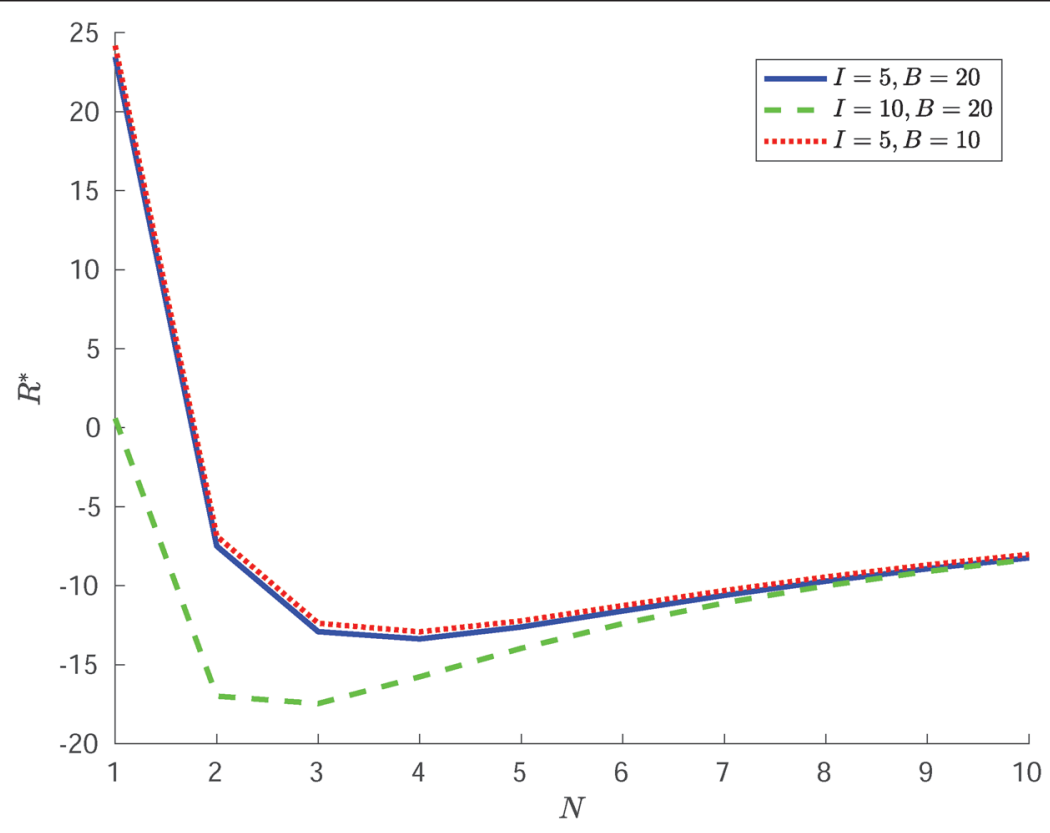

that is chosen by the profit-maximising storage operator is ambiguous). This is exactly because the incentives of the generating firms and the investment level of the profit-maximising storage operator are affected by the ramping charge. As discussed in Section 4, it is possible for an equilibrium with a profit-maximising storage operator in the presence of a ramping charge to yield greater social welfare than that attained by a welfare-maximising storage operator in the absence of a ramping charge. This is indeed observed with $N \geq 2$ firms in the figure. This result is because the ramping charge impacts generation quantities and the storage-investment decision. Indeed, for relatively low levels of market power, the improvement in social welfare occurs because the inclusion of the ramping charge is able to mimic the generation levels of the benchmark central-planning framework, in which no storage is deployed. Hence, with a large number of firms, both consumer and producer welfare increase in an equilibrium with a profit-maximising storage operator and a ramping charge $v i s-\grave{a}$-vis a welfare-maximising storage operator, while storage profit decreases and ramping payments are made to generators.

Figure 5 shows $R^{*}$, the equalising ramping charge. The figure shows that for a relatively low number of firms, i.e., in the presence of substantial market power in the generation sector, the ramping charge is positive. As discussed in Section 4, this positive ramping charge increases offpeak and decreases on-peak generation to reduce ramping. This increases the difference between on- and off-peak prices, which induces the profit-maximising storage operator to increase storage capacity (so it can sell a greater volume of energy and exploit the larger price difference).

The result is reversed with a relatively large number of firms, i.e., with less market power. In this case the profit maximiser's storage-investment level is above the welfare maximiser's. To correct this distorted investment, a negative ramping charge is imposed, i.e., generators are paid to ramp. As a result, off-peak generation decreases while on-peak generation increases, which decreases the difference between on- and off-peak prices. This decreased price difference reduces the ability of the profit-maximising storage operator to conduct intertemporal arbitrage and its incentives for storage investment. 
Figure 6: Profit and welfare maximisers' storage-investment levels and optimal storageinvestment level under centralised planning with linear marginal generation costs.

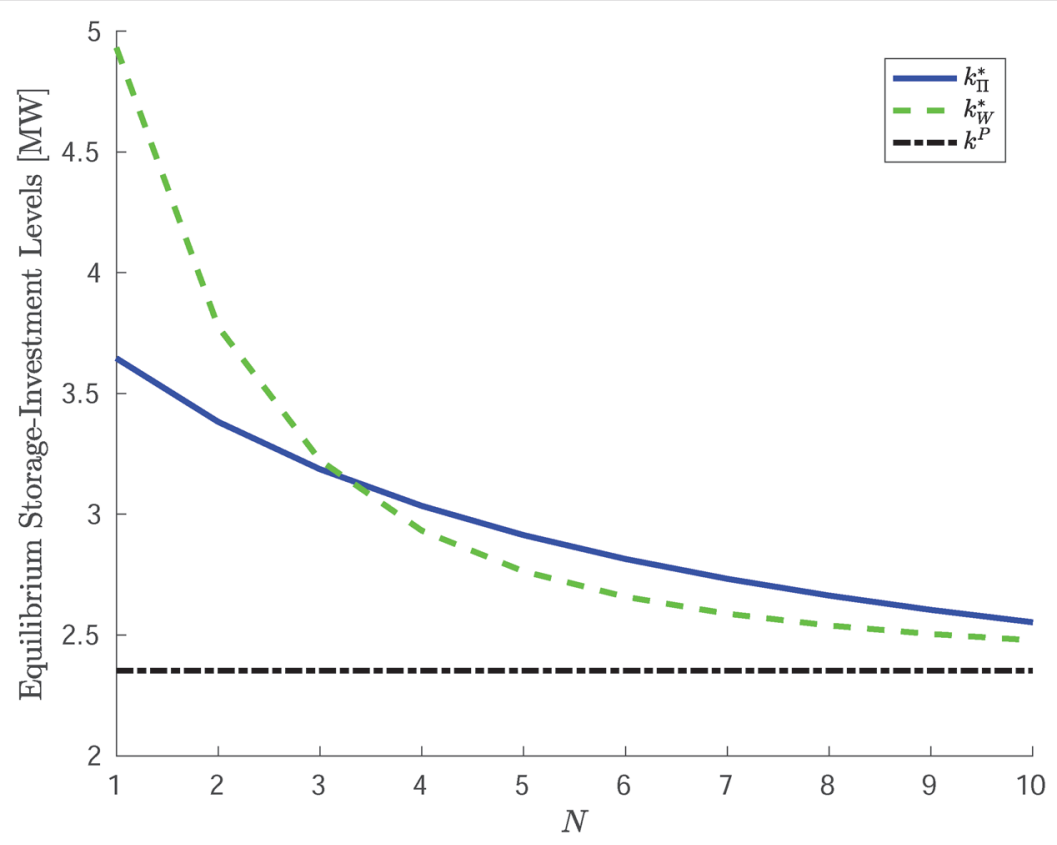

Figure 5 also illustrates the qualitative effects of higher generation or storage costs on the equalising ramping charge. The figure shows that increasing either cost reduces $R^{*}$. Intuitively, this is because more costly investment or operational decisions are more onerous to alter through the ramping charge.

\subsection{Results with Linear Marginal Generation Costs}

It is not possible to conduct the types of comparative statics for the case of linear marginal generation costs that we do for constant marginal generation costs in Section 3. We, instead, provide some numerical examples here (with the derivations of the underlying analytical expressions given in Appendix 7.2). These examples demonstrate that many of the qualitative properties of the equilibria that we have with constant marginal generation costs carry over to the linear case. We assume here that generator costs are given by:

$$
c\left(g_{n, t}\right)=B g_{n, t}+\frac{1}{2} N K g_{n, t}^{2}, n=1, \ldots, N
$$

We choose this parametric form for the generators' cost functions (specifically, the inclusion of $N$ in the quadratic term) to ensure that the sectoral perfectly competitive supply is equal regardless of the number of firms. Without such an adjustment, the industry's marginal cost function would vary with $N$. Such a framework would not allow for easy comparison of market outcomes across different levels of market power in the generation sector. We use the same base case parameter values that are summarised in Table 1, assuming that $K=1$.

Figure 6 summarises the profit and welfare maximisers' storage-investment levels with linear marginal generation costs. It also provides the optimal level of storage investment in a benchmark case in which there is a social planner that owns and operates all of the storage and generation 
Figure 7: Change in social welfare under different storage-investment equilibria relative to no-storage case with linear marginal generation costs

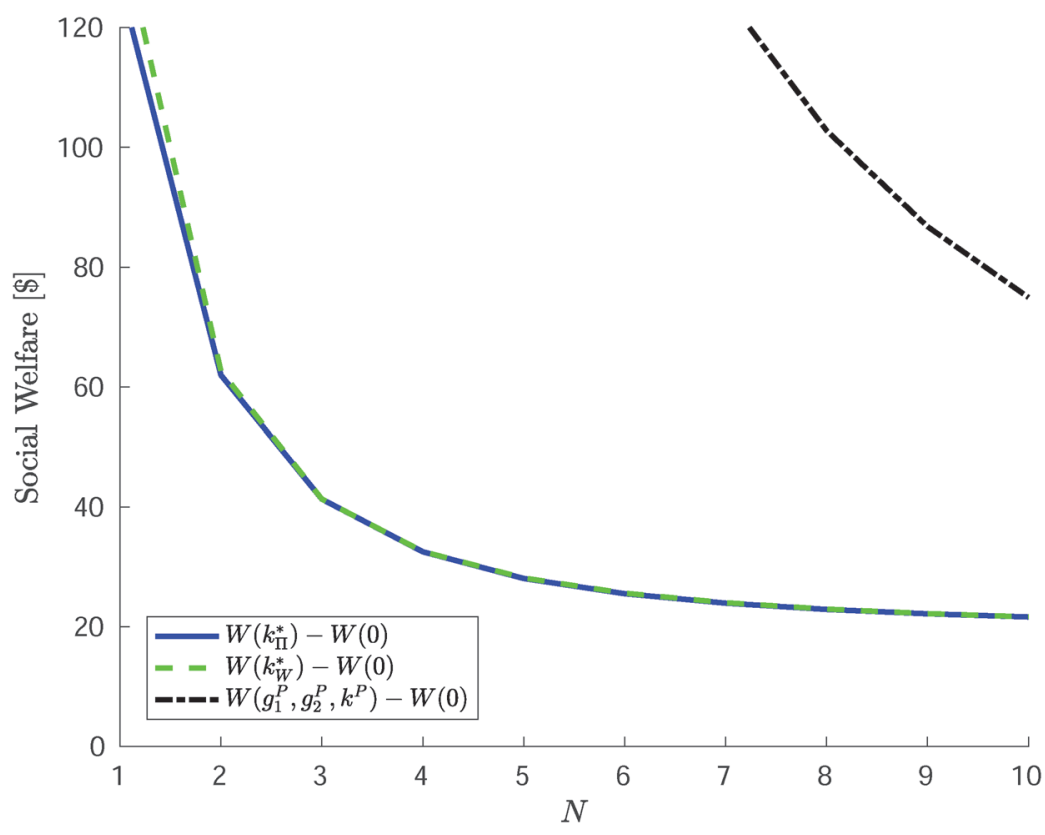

capacity. We retain the same notational conventions that are used in Sections 3-4 in denoting equilibrium investment levels under different market structures. The figure illustrates that Propositions 1 and 4 carry over from the case of constant marginal generation costs to linear costs. Specifically, we see that the welfare maximiser's storage-investment level is greater than the profit maximiser's for a small number of generating firms. The profit and welfare maximisers' storage-investment levels both decrease with the number of generating firms. Eventually, once there are a sufficient number of generating firms in the market, the profit-maximising storage operator overinvests in storage relative to the level of the welfare-maximising storage operator. One difference between the cases of constant and linear marginal generation costs is that some level of storage investment is optimal with centralised planning. This is because even if the generation sector is perfectly competitive, some price difference remains between the on- and off-peak periods. So long as the investment cost of storage is not unduly high or its roundtrip efficiency too low, it is socially beneficial to use some storage capacity to reduce this price difference.

Finally, Figure 7 shows the change in social welfare between different equilibrium storage-investment levels and a no-storage case. The figure shows that investing in some amount of storage capacity is always welfare enhancing vis-à-vis having no storage capacity. On the other hand, Proposition 5 and the second part of Proposition 1 do not hold (i.e., we do not observe cases with social welfare losses arising from the profit maximiser's storage-investment level). Indeed, with a sufficiently high linear marginal generation cost, there is no possibility of having 'too much' storage relative to the no-storage case.

\section{DISCUSSION AND CONCLUSIONS}

The past few years have seen growing interest in energy storage. This is driven by recognition of many applications for which storage can be used, the advent of restructured electricity 
markets, and the growing role of storage for integrating renewable energy sources. As such, energy storage is being championed by policymakers and is increasingly being installed by utilities, standalone merchant firms, and individual electricity customers. Despite this, the academic literature cautions that storage is not without its tradeoffs, insomuch as it could lead to reduced social welfare and increased $\mathrm{CO}_{2}$ emissions under certain market-structure conditions.

We contribute to the literature studying the welfare impacts of energy storage by examining the equilibrium storage-investment level under a variety of market structures. Our analytical results demonstrate that a welfare-maximising storage operator invests in more storage capacity than a profit-maximising firm only if the generation sector is relatively imperfectly competitive. In fact, if the generation sector is sufficiently competitive, then a profit-maximising storage investor may degrade social welfare compared to a no-storage case. This finding stands in contrast to that of Sioshansi (2014), who shows that storage cannot yield welfare losses with a perfectly competitive generation sector if only the operation (but not the investment) decision is endogenised. We find that a ramping charge on generation can induce the profit-maximising storage operator to invest in the welfare maximiser's storage-investment level. Indeed, we find that the equalising ramping charge may yield social welfare that is greater than that attained by the welfare maximiser's storage-investment level, because the ramping charge can mitigate market failures in a relatively uncompetitive generation sector. Our numerical examples illustrate these principles and show that many of them carry over to a more realistic case with linear marginal generation costs. Hence, the policy insights that stem from our analysis can be used by regulatory bodies to align better the incentives of a profit-maximising storage operator with those of society.

By taking a stylised approach, we are able to unpick methodically the countervailing incentives driving storage investment, e.g., the tradeoff between profit margin and trading volume. Nevertheless, in doing so, we omit some pertinent real-world details that may lead to a comprehensive comparison of storage investment and use under different market structures in future work. For example, uncertainty (pertaining either to load or renewable generation) could make the default storage operations of charging during off-peak periods and discharging during on-peak periods more risky, thereby necessitating a stochastic- or robust-optimisation model. Likewise, storage siting is important in alleviating transmission congestion. Towards this end, our two-period model could be enhanced by incorporating two nodes and a potentially congested transmission line. Finally, a complete tri-level model, e.g., following the structure that is employed by Murphy and Smeers (2010), with the policymaker at the upper-most level, storage investment at the middle level, and market operations at the lowest level could be implemented to identify optimal policies (e.g., ramping charges) and their interaction with storage adoption in the presence of imperfect competition.

Our analysis also neglects some market-structure realities. We assume a single storage operator, constant marginal generation costs, and two operating periods to enable deriving closed-form expressions characterising market equilibria. Relaxing any of these assumptions may significantly complicate the derivations or may necessitate the use of computational techniques. Future work may apply such techniques (e.g., a case with multiple storage operators would render an equilibrium problem subject to equilibrium constraints) to numerical examples and discern results from there. Sioshansi (2014) does analyse a case of storage owned by multiple generating firms and concludes that there can be mixed welfare outcomes, depending on the underlying market structure. Some of these results may extend to a case in which storage-investment decisions are endogenised. Our modelling framework could also be extended, if a purely computational approach is taken, to account for other services being provided by storage. Sioshansi (2011); Green and Vasilakos (2012); Mauritzen 
(2013) examine the use of storage for wind integration. Such an analysis may reveal further sources of market inefficiencies.

Our analysis also abstracts away the question of who the welfare-maximising storage operator is. In theory, this could be a case in which storage is built and operated by the market operator or independent system operator (ISO). However, this may be untenable in practice because a market operator or ISO that owns storage would no longer be an independent third party (which is an important concept underlying wholesale electricity-market restructuring). Sioshansi (2017) notes that a merchant energy storage developer proposed having an ISO directly control the dispatch of a pumped hydroelectric storage plant to mitigate transmission congestion. A contentious issue that was raised in the ensuing case surrounded the impact that the proposal would have on the market independence of the ISO. In the end, the Federal Energy Regulatory Commission (FERC) denied the request, as it determined that the impact on the independence of the ISO outweighed any co-ordination benefit of having the ISO dispatch the plant. ${ }^{1}$

Our analysis has important real-world policy and regulatory implications. Our finding that profit-maximising storage investment is not welfare maximising means that policymakers and regulators should be cognisant of what impacts energy storage can have within particular markets. These impacts will be dependent on the relative competitiveness of the generation sector. The fact that a sufficiently competitive generation sector can result in net welfare losses (compared to a no-storage case) should be cause for concern. This can be especially true as energy storage is first adopted within a market, because there may be one dominant storage operator, as we assume in our modelling framework. Regulatory decision making with respect to the market efficiency impacts of energy storage has been somewhat muted to date. For instance, in its recent order on integrating energy storage into electricity markets (cf. Electric Storage Participation in Markets Operated by Regional Transmission Organizations and Independent System Operators, Order Number 841), the FERC largely sidesteps the issue of the market impacts of energy storage. Instead, it implicitly assumes that energy storage does not have deleterious effects on market efficiency. Our findings suggest that such a view may be incorrect, depending on other factors related to the underlying structure of the market.

\section{ACKNOWLEDGMENTS}

A. Siddiqui has been supported by funding received from the Social Sciences and Humanities Research Council of Canada under grant number 435-2017-0068. We have benefited from feedback received at the Institute for Operations Research and the Management Sciences Annual Meeting, the International Conference of the International Association for Energy Economics, seminars at the University of Oslo, the University of Guelph, Aalto University, and HEC Montréal, and from the editors and four anonymous reviewers. R. Sioshansi thanks A. Sorooshian for helpful conversations and suggestions. All remaining errors are our own.

\section{REFERENCES}

Denholm, P., E. Ela, B. Kirby, and M.R. Milligan (2010). “The Role of Energy Storage with Renewable Electricity Generation.” Tech. Rep. NREL/TP-6A2-47187, National Renewable Energy Laboratory, Golden, CO. https://doi.org/10.2172/972169. 
Dvorkin, Y., R. Fernández Blanco Carramolino, Y. Wang, B. Xu, D. Kirschen, H. Pandžić, J.-P. Watson, and C.A. Silva-Monroy (2018). "Co-planning of Investments in Transmission and Merchant Energy Storage." IEEE Transactions on Power Systems 33: 245-256. https://doi.org/10.1109/TPWRS.2017.2705187.

EPRI (1976). “Assessment of energy storage systems suitable for use by electric utilities.” Tech. Rep. EPRI-EM-264, Electric Power Research Institute, Palo Alto, CA, USA.

EPRI-DOE (2003). "EPRI-DOE Handbook for Energy Storage for Transmission and Distribution Applications.” Tech. Rep. 1001834, Electric Power Research Institute and the U.S. Department of Energy, Palo Alto, CA and Washington, DC, USA.

Eyer, J. M. and G. Corey, (2010). "Energy Storage for the Electricity Grid: Benefits and Market Potential Assessment Guide." Tech. Rep. SAND2010-0815, Sandia National Laboratories, Albuquerque, NM.

Eyer, J M., J.J. Iannucci, and P.C. Butler (2005). "Estimating Electricity Storage Power Rating and Discharge Duration for Utility Transmission and Distribution Deferral.” Tech. Rep. SAND2005-7069, Sandia National Laboratories, Albuquerque, NM.

Green, R. and N. Vasilakos (2012). "Storing Wind for a Rainy Day: What Kind of Electricity Does Denmark Export?" The Energy Journal 33: 1-22. https://doi.org/10.5547/01956574.33.3.1.

Mauritzen, J. (2013). "Dead Battery? Wind Power, the Spot Market, and Hydropower Interaction in the Nordic Electricity Market.” The Energy Journal 34: 103-123. https://doi.org/10.5547/01956574.34.1.5.

Murphy, F. and Y. Smeers (2010). "On the Impact of Forward Markets on Investments in Oligopolistic Markets with Reference to Electricity.” Operations Research 58: 515-528. https://doi.org/10.1287/opre.1090.0753.

Nasrolahpour, E., S.J. Kazempour, H. Zareipour, and W.D. Rosehart (2016). "Strategic Sizing of Energy Storage Facilities in Electricity Markets." IEEE Transactions on Sustainable Energy 7: 1462-1472. https://doi.org/10.1109/ TSTE.2016.2555289.

Nourai, A. (2007). "Installation of the First Distributed Energy Storage System (DESS) at American Electric Power (AEP)." Tech. Rep. SAND2007-3580, Sandia National Laboratories, Albuquerque, NM. https://doi.org/10.2172/912663.

Nourai, A., V.I. Kogan, and C.M. Schafer (2008). "Load Leveling Reduces T\&D Line Losses.” IEEE Transactions on Power Delivery 23: 2168-2173. https://doi.org/10.1109/TPWRD.2008.921128.

O’Dwyer, C. and D. Flynn (2015). “Using Energy Storage to Manage High Net Load Variability at Sub-Hourly Time-Scales." IEEE Transactions on Power Systems 30: 2139-2148. https://doi.org/10.1109/TPWRS.2014.2356232.

Schill, W.-P. and C. Kemfert (2011). "Modeling Strategic Electricity Storage: The Case of Pumped Hydro Storage in Germany." The Energy Journal 32: 59-88. https://doi.org/10.5547/ISSN0195-6574-EJ-Vol32-No3-3.

Shahmohammadi, A., R. Sioshansi, A.J. Conejo, and S. Afsharnia (2018a). "Market Equilibria and Interactions Between Strategic Generation, Wind, and Storage." Applied Energy 220: 876-892. https://doi.org/10.1016/j.apenergy.2017.10.035.

Shahmohammadi, A., R. Sioshansi, A.J. Conejo, and S. Afsharnia (2018b). "The Role of Energy Storage in Mitigating Ramping Inefficiencies Caused by Variable Renewable Generation.” Energy Conversion and Management 162: 307-320. https:// doi.org/10.1016/j.enconman.2017.12.054.

Sioshansi, R. (2010). "Welfare Impacts of Electricity Storage and the Implications of Ownership Structure." The Energy Journal 31: 173-198. https://doi.org/10.5547/ISSN0195-6574-EJ-Vol31-No2-7.

Sioshansi, R. (2011). "Increasing the Value of Wind with Energy Storage." The Energy Journal 32: 1-30. https://doi. org/10.5547/ISSN0195-6574-EJ-Vol32-No2-1.

Sioshansi, R. (2014). "When Energy Storage Reduces Social Welfare." Energy Economics 41: 106-116. https://doi. org/10.1016/j.eneco.2013.09.027.

Sioshansi, R. (May 2017). "Using Storage-Capacity Rights to Overcome the Cost-Recovery Hurdle for Energy Storage." IEEE Transactions on Power Systems 32: 2028-2040. https://doi.org/10.1109/TPWRS.2016.2607153.

Sioshansi, R., P. Denholm, and T. Jenkin (2012). "Market and Policy Barriers to Deployment of Energy Storage.” Economics of Energy \& Environmental Policy 1: 47-63. https://doi.org/10.5547/2160-5890.1.2.4.

Sioshansi, R., P. Denholm, T. Jenkin, and J. Weiss (2009). "Estimating the Value of Electricity Storage in PJM: Arbitrage and Some Welfare Effects.” Energy Economics 31: 269-277. https://doi.org/10.1016/j.eneco.2008.10.005.

Virasjoki, V., P. Rocha, A. S. Siddiqui, and A. Salo (2016). "Market Impacts of Energy Storage in a Transmission-Constrained Power System.” IEEE Transactions on Power Systems 31: 4108-4117. https://doi.org/10.1109/TPWRS.2015.2489462.

von Hirschhausen, C. (2014). "The German "Energiewende"-An Introduction." Economics of Energy \& Environmental Policy 3: 1-12. https://doi.org/10.5547/2160-5890.3.2.chir.

Zhao, B., A. J. Conejo, and R. Sioshansi (2017). "Unit Commitment Under Gas-Supply Uncertainty and Gas-Price Variability.” IEEE Transactions on Power Systems 32: 2394-2405. https://doi.org/10.1109/TPWRS.2016.2602659. 


\section{APPENDIX}

\subsection{Proofs of Lemmata, Propositions, and Corollary}

Proof of Lemma 1 We show that $g_{n, 1}=g_{m, 1}, \forall n, m=1, \ldots, N$. The result that $g_{n, 2}=g_{m, 2}, \forall n, m=1, \ldots, N$ can be shown analogously and is excluded for brevity. Because of the assumed properties of the inverse demand and cost functions, each generator's profit-maximisation problem is convex. Thus, the Karush-Kuhn-Tucker (KKT) conditions for $g_{n, 1}$, which are:

$$
\begin{aligned}
& -A_{1}+Z_{1} \cdot\left(g_{1}^{G}-F d\right)+Z_{1} g_{n, 1}+c^{\prime}\left(g_{n, 1}\right)-\mu_{n, 1}=0 \\
& 0 \leq \mu_{n, 1} \perp g_{n, 1} \geq 0,
\end{aligned}
$$

where $\mu_{n, 1}$ is the Lagrange multiplier associated with generator $n$ 's period-1 non-negativity constraint, are sufficient for a global optimum.

Subtracting condition (17) for generator $m$ from that for generator $n \neq m$ gives:

$$
Z_{1} \cdot\left(g_{n, 1}-g_{m, 1}\right)+c^{\prime}\left(g_{n, 1}\right)-c^{\prime}\left(g_{m, 1}\right)-\mu_{n, 1}+\mu_{m, 1}=0 \text {. }
$$

Suppose for contradiction that the production levels are not symmetric and without loss of generality that the generators are labelled such that $g_{n, 1}>g_{m, 1}$. From condition (18) we must have $\mu_{n, 1}=0$. Thus, (19) becomes:

$$
Z_{1} \cdot\left(g_{n, 1}-g_{m, 1}\right)+c^{\prime}\left(g_{n, 1}\right)-c^{\prime}\left(g_{m, 1}\right)+\mu_{m, 1}=0,
$$

which cannot hold because by assumption we have that $Z_{1} \cdot\left(g_{n, 1}-g_{m, 1}\right)>0$, by convexity of the cost function we have that $c^{\prime}\left(g_{n, 1}\right)-c^{\prime}\left(g_{m, 1}\right) \geq 0$, and the KKT conditions require that $\mu_{m, 1} \geq 0$. This gives the desired contradiction, which proves the result.

Proof of Lemma 2 Assume for contradiction that $\left(k^{*}, d^{*}\right)$ is an optimal solution in which $k^{*} \neq d^{*}$. By the inequality constraint in the problem, we must have $k^{*}>d^{*}$. Consider the alternate solution $\left(\tilde{k}, d^{*}\right)$, with:

$$
\tilde{k}=\frac{k^{*}+d^{*}}{2}
$$

This solution is clearly feasible in the problem constraint. Moreover, we have that:

$$
\left[\mathcal{P}\left(d^{*}\right)-\left(I k^{* 2}\right) / 2\right]-\left[\mathcal{P}\left(d^{*}\right)-\left(I \tilde{k}^{2}\right) / 2\right]=I \cdot\left(\tilde{k}^{2}-k^{* 2}\right) / 2<0,
$$

because by construction $\tilde{k}<k^{*}$, meaning that $\left(\tilde{k}, d^{*}\right)$ gives a smaller objective-function value than $\left(k^{*}, d^{*}\right)$, contradicting the optimality of $\left(k^{*}, d^{*}\right)$.

Proof of Proposition 1 To show the first part of the proposition, we compare the expressions in (7) and (12), which gives that for $k_{W}^{*}$ to be greater than or equal to $k_{\Pi}^{*}$ we must have:

$$
\frac{A_{2}-F A_{1}-B N \cdot(F-1)(N+2)}{I \cdot(N+1)^{2}+F^{2} Z_{1}+Z_{2}} \geq \frac{A_{2}-F A_{1}-B N \cdot(F-1)}{I \cdot(N+1)+2 F^{2} Z_{1}+2 Z_{2}},
$$

which simplifies to: 


$$
\begin{aligned}
Q(N) & =-N^{2} \cdot\left[I \cdot\left(A_{2}-F A_{1}\right)+I B \cdot(F-1)+2\left(F^{2} Z_{1}+Z_{2}\right) B \cdot(F-1)\right] \\
& -N \cdot\left[I \cdot\left(A_{2}-F A_{1}\right)+I B \cdot(F-1)+3\left(F^{2} Z_{1}+Z_{2}\right) B \cdot(F-1)\right]+\left(A_{2}-F A_{1}\right)\left(F^{2} Z_{1}+Z_{2}\right) \geq 0 .
\end{aligned}
$$

Because the coefficients of $N$ and $N^{2}$ in $Q(N)$ are both negative, we have $Q^{\prime \prime}(N)<0, \forall N$ and $Q^{\prime}(0)<0$. Thus, $Q(N)$ is a downward-facing parabola. Furthermore, because $Q(0)>0, Q(N)$ has a unique positive root, $\bar{N}$. Hence, for $N<\bar{N}$, we have $k_{W}^{*} \geq k_{\Pi}^{*}$, otherwise, $k_{W}^{*}<k_{\Pi}^{*}$.

To show the second part of the proposition, we note that from (10) we have that:

$$
\begin{aligned}
W^{\prime}(k)=F \frac{Z_{1}}{(N+1)^{2}}\left[N \frac{A_{1}-B}{Z_{1}}+(2 N+1) F k\right] & +\frac{Z_{2}}{(N+1)^{2}}\left[(2 N+1) k-N \frac{A_{2}-B}{Z_{2}}\right] \\
& +\left(k_{\Pi}^{*}-k\right) \frac{I \cdot(N+1)+2 F^{2} Z_{1}+2 Z_{2}}{N+1} .
\end{aligned}
$$

Substituting $k_{\Pi}^{*}$ into (20) gives:

$$
\begin{aligned}
W^{\prime}\left(k_{\Pi}^{*}\right) & =F \frac{Z_{1}}{(N+1)^{2}}\left[N \frac{A_{1}-B}{Z_{1}}+(2 N+1) F k_{\Pi}^{*}\right]+\frac{Z_{2}}{(N+1)^{2}}\left[(2 N+1) k_{\Pi}^{*}-N \frac{A_{2}-B}{Z_{2}}\right] \\
& =\frac{Q(N)}{(N+1)^{2}\left[I \cdot(N+1)+2\left(F^{2} Z_{1}+Z_{2}\right)\right]} .
\end{aligned}
$$

The denominator, $(N+1)^{2}\left[I \cdot(N+1)+2\left(F^{2} Z_{1}+Z_{2}\right)\right]$, is strictly positive. Thus, the only way for $W^{\prime}\left(k_{\Pi}^{*}\right)$ to be positive (negative) is if $N<\bar{N}(N>\bar{N})$.

Proof of Proposition $2 \quad \bar{N}$ is defined as the root of the characteristic polynomial (cf. Proposition 1):

$$
Q(\bar{N})=0 .
$$

To show the first part of the proposition, we totally differentiate this defining equation with respect to $I$, which gives:

$$
\frac{\partial}{\partial I} Q(\bar{N})+\frac{\partial}{\partial N} Q(\bar{N}) \frac{\partial \bar{N}}{\partial I}=0 .
$$

This can be rewritten as:

$$
\frac{\partial \bar{N}}{\partial I}=-\frac{\frac{\partial}{\partial I} Q(\bar{N})}{\frac{\partial}{\partial N} Q(\bar{N})} .
$$

We have:

$$
\frac{\partial}{\partial I} Q(\bar{N})=-\left(\bar{N}^{2}+\bar{N}\right)\left[A_{2}-F A_{1}+B \cdot(F-1)\right]<0,
$$

and we also know (cf. the proof of Proposition 1) that $Q^{\prime \prime}(N)<0, \forall N$ and $Q^{\prime}(0)<0$, meaning that:

$$
\frac{\partial}{\partial N} Q(\bar{N})<0
$$


Thus, from (21) we have that:

$$
\frac{\partial \bar{N}}{\partial I}<0
$$

which is the desired result.

To show the second part of the proposition, we totally differentiate $Q(\bar{N})=0$ with respect to $B$, which gives:

$$
\frac{\partial}{\partial B} Q(\bar{N})+\frac{\partial}{\partial N} Q(\bar{N}) \frac{\partial \bar{N}}{\partial B}=0
$$

and which can be rewritten as:

$$
\frac{\partial \bar{N}}{\partial B}=-\frac{\frac{\partial}{\partial B} Q(\bar{N})}{\frac{\partial}{\partial N} Q(\bar{N})} .
$$

We have that:

$$
\frac{\partial}{\partial B} Q(\bar{N})=-I \cdot(F-1) \bar{N} \cdot(\bar{N}+1)-\left(F^{2} Z_{1}+Z_{2}\right)(F-1) N \cdot(2 N+3)<0,
$$

and we know that:

$$
\frac{\partial}{\partial N} Q(\bar{N})<0
$$

Thus, we have:

$$
\frac{\partial \bar{N}}{\partial B}<0
$$

which is the desired result.

Proof of Proposition 3 To show the impact of storage use on the price differential, we note that from (3) and (4) we have:

$$
p_{2}(d)-F p_{1}(d)=\frac{A_{2}-F A_{1}-B N \cdot(F-1)-d \cdot\left(Z_{2}+F^{2} Z_{1}\right)}{N+1} .
$$

The coefficient on $d$ :

$$
-\frac{Z_{2}+F^{2} Z_{1}}{N+1}
$$

is negative, meaning that the price differential decreases with storage use.

To show the impact of the number of generating firms on the price differential, we partially differentiate (22) with respect to $N$, which gives:

$$
\frac{\partial}{\partial N}\left(p_{2}(d)-F p_{1}(d)\right)=-\frac{\left[p_{2}(d)-F p_{1}(d)+B \cdot(F-1)\right]}{N+1} .
$$

This partial derivative is negative. Thus, it follows that the price differential decreases with $N$. 
Proof of Proposition 4 To, first, show the effect of the number of generating firms on the profit maximiser's storage-investment level, we partially differentiate (7) with respect to $N$, which gives:

$$
\frac{\partial}{\partial N} k_{\Pi}^{*}=-\frac{\left[A_{2}-F A_{1}-B N \cdot(F-1)\right] I}{\left(I \cdot(N+1)+2 F^{2} Z_{1}+2 Z_{2}\right)^{2}}-\frac{B \cdot(F-1)}{I \cdot(N+1)+2 F^{2} Z_{1}+2 Z_{2}} .
$$

This partial derivative is strictly negative. Thus, it follows that the profit maximiser's storage-investment level decreases with the number of generating firms.

Next, to show the impact of the number of firms on the welfare maximiser's storage-investment level, we partially differentiate (12) with respect to $N$, giving:

$$
\frac{\partial}{\partial N} k_{W}^{*}=-\frac{2\left[A_{2}-F A_{1}-B N \cdot(F-1)(N+2)\right] I \cdot(N+1)}{\left(I \cdot(N+1)^{2}+F^{2} Z_{1}+Z_{2}\right)^{2}}-\frac{2 B \cdot(F-1)(N+1)}{I \cdot(N+1)^{2}+F^{2} Z_{1}+Z_{2}} .
$$

This partial derivative is strictly negative, from which it follows that the welfare maximiser's storage-investment level decreases with the number of generating firms.

Proof of Proposition 5 We have:

$$
\begin{aligned}
W\left(k_{\Pi}^{*}\right)- & W(0)=-\frac{1}{2} I B \cdot(F-1) N^{3} \\
& -\left[2 B \cdot(F-1)\left(F^{2} Z_{1}+Z_{2}\right)+I \cdot\left(\frac{1}{2}\left(A_{2}-F A_{1}\right)+2 B(F-1)\right)\right] N^{2} \\
& -\left[B \cdot(F-1)\left(\frac{3}{2} I+\frac{7}{2}\left(F^{2} Z_{1}+Z_{2}\right)\right)\right] N+\frac{1}{2}\left(A_{2}-F A_{1}\right)\left[I+3\left(F^{2} Z_{1}+Z_{2}\right)\right]=S(N) .
\end{aligned}
$$

$S(N)$ is a cubic polynomial, which is strictly positive and decreasing at $N=0 . S(N)$, therefore, has exactly one positive root and either zero or two negative roots. Let $\tilde{N}$ denote the positive root of $S(N) . \tilde{N}$ is the critical number of firms, above which no storage yields higher social welfare than the profit maximiser's storage-investment level.

Proof of Corollary $1 W\left(k_{\Pi}^{*}\right)<W(0)$ means that an infinitesimal increase in the storage-investment level from $k_{\Pi}^{*}$ decreases social welfare. From Proposition 1, we know that this outcome is possible only when the number of firms is greater than $\bar{N}$. Hence, we must have that $\tilde{N}>\bar{N}$ when $W\left(k_{\Pi}^{*}\right)<W(0)$.

\subsection{Market Equilibria with Linear Marginal Generation Costs}

Here, we investigate how storage investment is affected by linear marginal generation costs. All modelling assumptions are the same as those that are in Sections 2-4, with the exception of the generation cost. We now assume that generation costs have the quadratic form:

$$
c\left(g_{n, t}\right)=B g_{n, t}+\frac{1}{2} N K g_{n, t}^{2}, n=1, \ldots, N,
$$

where $B, K>0$.

We proceed with this analysis in four steps. We first derive the equilibrium production levels of the generating firms in the two operating periods. Next, we determine equilibrium storage-operation and -investment decisions for the profit- and welfare-maximising storage operators, 
respectively. Finally, we find the benchmark generation and storage-related decisions if all of them are made by a single social planner.

\subsubsection{Generator Equilibrium}

With linear marginal generation costs, generator $n$ 's profit-maximisation problem becomes:

$$
\max _{g_{n, 1}, g_{n, 2} \geq 0} P_{1}\left(g_{1}^{G}-F d\right) g_{n, 1}-B g_{n, 1}-\frac{1}{2} N K g_{n, 1}^{2}+P_{2}\left(g_{2}^{G}+d\right) g_{n, 2}-B g_{n, 2}-\frac{1}{2} N K g_{n, 2}^{2} \text {. }
$$

Because this is a convex optimisation problem, its KKT conditions, which are:

$$
0 \leq-A_{1}+Z_{1} \cdot\left(g_{1}^{G}-F d\right)+Z_{1} g_{n, 1}+B+N K g_{n, 1} \perp g_{n, 1} \geq 0,
$$

and:

$$
0 \leq-A_{2}+Z_{2} \cdot\left(g_{2}^{G}+d\right)+Z_{2} g_{n, 2}+B+N K g_{n, 2} \perp g_{n, 2} \geq 0
$$

are sufficient for a global optimum. We can appeal to Lemma 1 to conclude that the equilibrium production levels of the generators are symmetric in each of the two periods. Adding the assumption that we have an interior solution (otherwise we have $g_{t}^{G}=0$ in at least one period, which is an uninteresting case), gives:

$$
g_{1}^{G}(d)=\frac{N \cdot\left(A_{1}+Z_{1} F d-B\right)}{L_{1}},
$$

and:

$$
g_{2}^{G}(d)=\frac{N \cdot\left(A_{2}-Z_{2} d-B\right)}{L_{2}}
$$

as the aggregate production levels of the generators in the two periods, where we define:

$$
L_{1}=Z_{1} \cdot(N+1)+N K,
$$

and:

$$
L_{2}=Z_{2} \cdot(N+1)+N K
$$

We finally substitute these aggregate production levels into the period- 1 and- 2 inverse demand function to obtain equilibrium prices:

$$
p_{1}(d)=\frac{\left(A_{1}+Z_{1} F d\right)\left(Z_{1}+N K\right)+Z_{1} B N}{L_{1}},
$$

and:

$$
p_{2}(d)=\frac{\left(A_{2}-Z_{2} d\right)\left(Z_{2}+N K\right)+Z_{2} B N}{L_{2}},
$$

in the two periods. 


\subsubsection{Investment and Operating Equilibrium of Profit-Maximising Storage Operator}

We analyse the behaviour of a profit-maximising storage operator by first examining its operating decisions, which are governed by the profit-maximisation problem:

$$
\begin{array}{ll}
\max _{d} & d \cdot\left[p_{2}(d)-F p_{1}(d)\right] \\
\text { s.t. } & 0 \leq d \leq k, \quad(\mu)
\end{array}
$$

where $\mu$ is the Lagrange multiplier associated with the storage-capacity constraint and we use (25) and (26) as the prices in the two periods. Because $p_{1}(d)$ and $p_{2}(d)$ depend on $d$ linearly, this profit-maximisation is convex and its KKT conditions are necessary and sufficient for a global optimum. This storage operator's problem has the KKT conditions:

$$
\begin{aligned}
& 0 \leq-\left(p_{2}(d)-F p_{1}(d)\right)-d p_{2}^{\prime}(d)+d F p_{1}^{\prime}(d)+\mu \perp d \geq 0, \\
& 0 \leq k-d \perp \mu \geq 0,
\end{aligned}
$$

which yields the same solution that is given in (5), with the caveat that we use the price functions that are given by (25) and (26), as opposed to (3) and (4).

Turning to the storage operator's investment decision, this is determined by the profit-maximisation problem:

$$
\max _{k}\left[p_{2}(k)-F p_{1}(k)\right] k-\left(I k^{2}\right) / 2
$$

where by Lemma 2 we know that storage is fully utilised in the operating stage, meaning that $d=k$. We further assume that we have an interior solution, in which storage capacity is built (i.e., that the non-negativity constraint, $k \geq 0$, is non-binding). The sufficient KKT condition for this problem is:

$$
-p_{2}(k)+F p_{1}(k)+I k-k p_{2}^{\prime}(k)+F k p_{1}^{\prime}(k)=0 .
$$

Substituting (25) and (26), respectively, for the periods-1 and -2 price functions and solving gives:

$$
k_{\Pi}^{*}=\frac{L_{1} \cdot\left[A_{2} \cdot\left(Z_{2}+N K\right)+Z_{2} B N\right]-F L_{2} \cdot\left[A_{1} \cdot\left(Z_{1}+N K\right)+Z_{1} B N\right]}{I L_{1} L_{2}+2 F^{2} Z_{1} L_{2} \cdot\left(Z_{1}+N K\right)+2 Z_{2} L_{1} \cdot\left(Z_{2}+N K\right)},
$$

as the profit maximiser's storage-investment level.

\subsubsection{Investment and Operating Equilibrium of Welfare-Maximising Storage Operator}

We analyse the case of a welfare-maximising storage operator in the same way that we do in the case of constant marginal generation costs. We begin by first deriving expressions for periods-1 and -2 consumer welfare, which are:

$$
W_{1}^{C}(k)=\int_{0}^{g_{1}^{G}(k)-F k}\left[P_{1}(x)-p_{1}(k)\right] d x=\frac{1}{2} Z_{1} \cdot\left[g_{1}^{G}(k)-F k\right]^{2},
$$

and:

$$
W_{2}^{C}(k)=\int_{0}^{g_{2}^{G}(k)+k}\left[P_{2}(x)-p_{2}(k)\right] d x=\frac{1}{2} Z_{2} \cdot\left[g_{2}^{G}(k)+k\right]^{2},
$$


respectively. These expressions are all written as functions of $k$, because we know from Lemma 2 that $d=k$ in a welfare-maximising equilibrium. Periods- 1 and -2 producer welfare are, similarly:

$$
W_{1}^{G}(k)=g_{1}^{G}(k) \cdot\left[p_{1}(k)-B-\frac{1}{2} K g_{1}^{G}(k)\right]=\frac{2 Z_{1}+N K}{2 N} g_{1}^{G}(k)^{2},
$$

and:

$$
W_{2}^{G}(k)=g_{2}^{G}(k) \cdot\left[p_{2}(k)-B-\frac{1}{2} K g_{2}^{G}(k)\right]=\frac{2 Z_{2}+N K}{2 N} g_{2}^{G}(k)^{2},
$$

respectively. The welfare of the storage operator is given by:

$$
W^{S}(k)=\left(2 k_{\Pi}^{*}-k\right) k \frac{I L_{1} L_{2} / 2+F^{2} Z_{1} L_{2} \cdot\left(Z_{1}+N K\right)+Z_{2} L_{1}\left(Z_{2}+N K\right)}{L_{1} L_{2}},
$$

where $k_{\Pi}^{*}$ is the value that is given by (27). Thus, substituting (23) and (24) for $g_{1}^{G}(d)$ and $g_{2}^{G}(d)$, we have:

$$
\begin{aligned}
W^{\prime}(k)= & F Z_{1} \frac{L_{2}^{2} \cdot\left\{Z_{1} N \cdot\left(A_{1}-B\right)+F \cdot\left[Z_{1}^{2}(2 N+1)+Z_{1} N K \cdot(N+2)+N^{2} K^{2}\right] k\right\}}{L_{1}^{2} L_{2}^{2}} \\
& +Z_{2} \frac{L_{1}^{2} \cdot\left[-Z_{2} N \cdot\left(A_{2}-B\right)+\left[Z_{2}^{2}(2 N+1)+Z_{2} N K \cdot(N+2)+N^{2} K^{2}\right] k\right]}{L_{1}^{2} L_{2}^{2}} \\
& +\left(k_{\Pi}^{*}-k\right)\left\{I+2 \frac{\left[F^{2} Z_{1} L_{1} L_{2}^{2} \cdot\left(Z_{1}+N K\right)+Z_{2} L_{1}^{2} L_{2} \cdot\left(Z_{2}+N K\right)\right]}{L_{1}^{2} L_{2}^{2}}\right\} .
\end{aligned}
$$

Assuming that we have an interior solution (i.e., that $k>0$ ), the investment problem can be written as:

$$
\max _{k}\left[W_{1}^{C}(k)-W_{1}^{C}(0)\right]+\left[W_{2}^{C}(k)-W_{2}^{C}(0)\right]+\left[W_{1}^{G}(k)-W_{1}^{G}(0)\right]+\left[W_{2}^{G}(k)-W_{2}^{G}(0)\right]+W^{S}(k),
$$

which is a convex quadratic program. Using (28), the sufficient KKT condition gives:

$$
k_{W}^{*}=\frac{A_{2} L_{1}^{2} J_{2}-F A_{1} L_{2}^{2} J_{1}+B N \cdot\left[Z_{2} L_{1}^{2} \cdot\left(L_{2}+Z_{2}\right)-F Z_{1} L_{2}^{2} \cdot\left(L_{1}+Z_{1}\right)\right]}{I L_{1}^{2} L_{2}^{2}+F^{2} Z_{1} L_{2}^{2} \cdot\left[Z_{1}^{2}+Z_{1} \kappa+N^{2} K^{2}\right]+Z_{2} L_{1}^{2} \cdot\left[Z_{2}^{2}+Z_{2} \kappa+N^{2} K^{2}\right]},
$$

as the welfare maximiser's storage-investment level, where:

$$
\begin{aligned}
& J_{1}=\left(Z_{1}+N K\right)^{2}+Z_{1} N^{2} K, \\
& J_{2}=\left(Z_{2}+N K\right)^{2}+Z_{2} N^{2} K,
\end{aligned}
$$

and:

$$
\kappa=N K \cdot(N+2) .
$$




\subsubsection{Benchmark Central Planner's Problem}

We know from Section 4, and (16) in particular, that building storage is suboptimal for a social planner with constant marginal generation costs. This may not be the case, however, with linear marginal generation costs. As a benchmark, we consider the following problem:

$$
\max _{g_{1}, g_{2}, k \geq 0} A_{1} \cdot\left(g_{1}-F k\right)-\frac{Z_{1}}{2}\left(g_{1}-F k\right)^{2}+A_{2} \cdot\left(g_{2}+k\right)-\frac{Z_{2}}{2}\left(g_{2}+k\right)^{2}-B \cdot\left(g_{1}+g_{2}\right)-\frac{K}{2}\left(g_{1}^{2}+g_{2}^{2}\right)-\frac{1}{2} I k^{2},
$$

in which a welfare-maximising central planner owns and operates all generation and storage facilities. This is a convex quadratic optimisation problem, thus its KKT conditions are sufficient for a global optimum. Assuming an interior solution (i.e., that generation levels are non-zero in both periods and that some energy storage is built), the KKT conditions give the optimal solution:

$$
\begin{aligned}
& g_{1}^{P}=\frac{A_{1}-B}{Z_{1}+K}+\frac{F Z_{1}}{Z_{1}+K} \cdot \frac{K \cdot\left[A_{2} \cdot\left(Z_{1}+K\right)-F A_{1} \cdot\left(Z_{2}+K\right)\right]-B \cdot\left[Z_{1} Z_{2} \cdot(F-1)-K \cdot\left(Z_{2}-F Z_{1}\right)\right.}{I \cdot\left(Z_{1}+K\right)\left(Z_{2}+K\right)+K \cdot\left[Z_{2} \cdot\left(Z_{1}+K\right)+F^{2} Z_{1} \cdot\left(Z_{2}+K\right)\right]}, \\
& g_{2}^{P}=\frac{A_{2}-B}{Z_{2}+K}-\frac{Z_{2}}{Z_{2}+K} \cdot \frac{K \cdot\left[A_{2} \cdot\left(Z_{1}+K\right)-F A_{1} \cdot\left(Z_{2}+K\right)\right]-B \cdot\left[Z_{1} Z_{2} \cdot(F-1)-K \cdot\left(Z_{2}-F Z_{1}\right)\right]}{I \cdot\left(Z_{1}+K\right)\left(Z_{2}+K\right)+K \cdot\left[Z_{2} \cdot\left(Z_{1}+K\right)+F^{2} Z_{1} \cdot\left(Z_{2}+K\right)\right]},
\end{aligned}
$$

and:

$$
k^{P}=\frac{K \cdot\left[A_{2} \cdot\left(Z_{1}+K\right)-F A_{1} \cdot\left(Z_{2}+K\right)\right]-B \cdot\left[Z_{1} Z_{2} \cdot(F-1)-K \cdot\left(Z_{2}-F Z_{1}\right)\right]}{I \cdot\left(Z_{1}+K\right)\left(Z_{2}+K\right)+K \cdot\left[Z_{2} \cdot\left(Z_{1}+K\right)+F^{2} Z_{1} \cdot\left(Z_{2}+K\right)\right]} .
$$

$k^{P}$ is positive so long as the linear portion of the marginal generation cost is relatively high, i.e., if $K \cdot\left(g_{2}^{P}-F g_{1}^{P}\right)>B \cdot(F-1)$. Hence, due to the linear marginal generation cost, storage may be required even under central planning because it enables the substitution of relatively inexpensive generation that is stored off-peak to displace higher-cost generation in the on-peak period. 


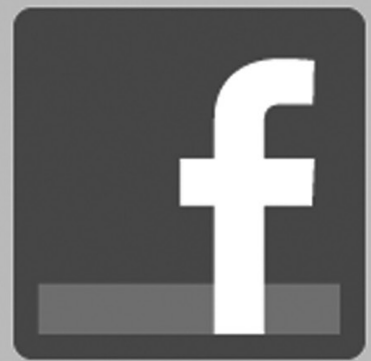

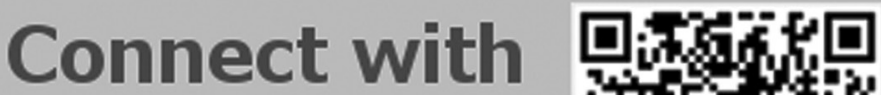

